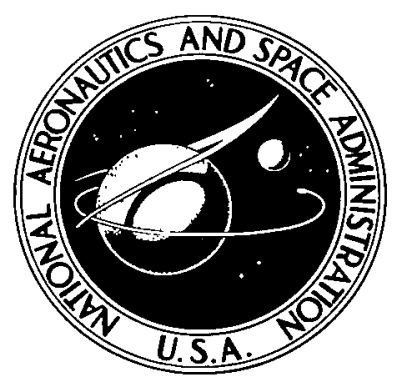

NASA TN D-8114

$\frac{⿱}{5}$
$\vdots$
$\vdots$
$z$
$\vdots$
$\vdots$

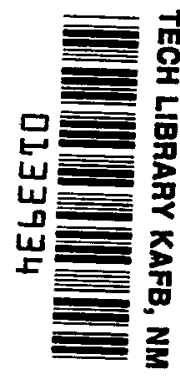

LOAN COPY: RETUIRN TO AFWIL TECHNICAL LIBRARY KIRTLAND AFB, N. M.

\title{
CHARACTERISTICS OF THE NASA LEWIS BUMPY-TORUS PLASMA GENERATED WITH POSITIVE APPLIED POTENTIALS
}

J. Reece Roth, Glenn A. Gerdin, and Richard W. Richardson

Lewis Research Center

Cleveland, Obio 44135

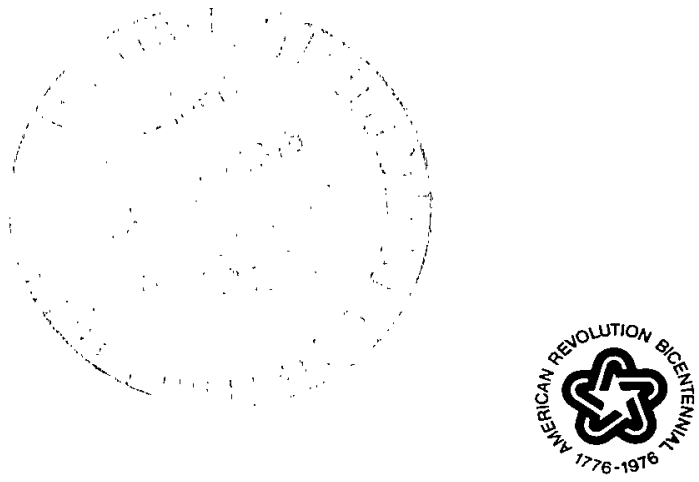

NATIONAL AERONAUTICS AND SPACE ADMINISTRATION - WASHINGTON, D. C. - MARCH 1976 
1. Report No.

NASA TN D-8114

4. Title and Subtitle

CHARACTERISTICS OF THE NASA LEWIS BUMPY-TORUS PLASMA GENERATED WITH POSITIVE APPLIED POTENTIALS

7. Author(s) J. Reece Roth, Lewis Research Center; and Glenn A. Gerdin and Richard W. Richardson, both NASA-NRC Postdoctoral Resident Research Associates

9. Performing Organization Name and Address

Lewis Research Center

National Aeronautics and Space Administration

Cleveland, Ohio 44135

12. Sponsoring Agency Name and Address

National Aeronautics and Space Administration

Washington, D. C. 20546

15. Supplementary Notes

16. Abstract

Experimental observations have been made during steady-state operation of the NASA Lewis bumpy-torus plasma at input powers up to $150 \mathrm{~kW}$ in deuterium and helium gas and with positive potentials applied to the midplane electrodes. In this steady-state ion heating method a modified Penning discharge is operated in a bumpy-torus confinement geometry such that the plasma is acted upon by a combination of strong electric and magnetic fields. Experimental investigation of a deuterium plasma revealed electron temperatures from 14 to $140 \mathrm{eV}$ and ion kinetic temperatures from 160 to $1785 \mathrm{eV}$. At least two distinct modes of operation exist, each of which is associated with a characteristic range of background pressure and electron temperature and each of which has a characteristic radial profile of electron temperature and number density. Experimental data show that the average ion residence time in the plasma is virtually independent of the magnetic field strength. Data were taken in the "standard" configuration, in which all 12 anode rings were at high voltage. Data were also taken in other symmetric configurations in which the toroidal plasma was generated by applying positive potentials to six anode rings, three anode rings, and a single anode ring, respectively.

17. Key Words (Suggested by Author(s))

Plasma heating; Hot ions; Plasma confinement; Bumpy torus; Plasma diffusion
18. Distribution Statement

Unclassified - unlimited

STAR Category 75 (rev.)
19. Security Classif. (of this report)

Unclassified

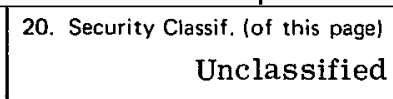

Unclassified
21. No. of Pages

55

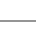

* For sale by the National Technical Information Service, Springfield, Virginia 22161 



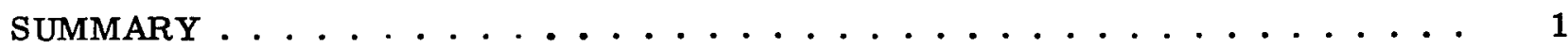

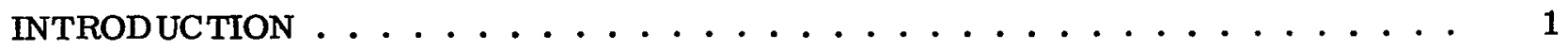

DESCRIPTION OF NASA LEWIS BUMPY -TORUS FACILITY $\ldots \ldots \ldots \ldots$

CHARACTERISTICS OF BUMPY-TORUS PLASMA $\ldots \ldots \ldots \ldots$

OPERATING REGIMES OF BUMPY-TORUS PLASMA . . . . . . . . . . . 10

ION HEATING IN THE BUMPY TORUS $\ldots \ldots \ldots \ldots \ldots \ldots \ldots$

RADIAL PROFILES OF FLOATING POTENTIAL $\ldots \ldots \ldots \ldots \ldots$

SPECTROSCOPICALLY DETERMINED RADIAL PROFILES OF ELECTRON

DENSITY AND TEMPERATURE $\ldots \ldots \ldots \ldots \ldots \ldots$

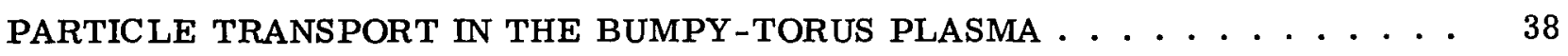

EFFECT ON PLASMA CHARACTERISTICS OF VARYING THE NUMBER OF

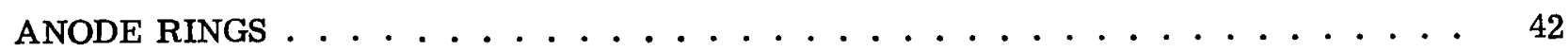

DISCUSSION AND CONCLUSIONS $\ldots \ldots \ldots \ldots \ldots \ldots \ldots$

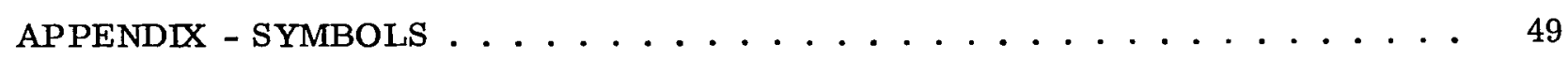

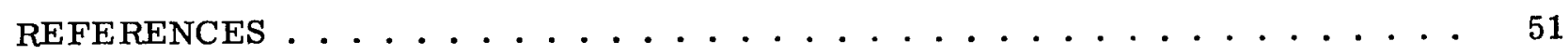




\title{
CHARACTERISTICS OF THE NASA LEWIS BUMPY-TORUS PLASMA GENERATED WITH POSITIVE APPLIED POTENTIALS
}

\author{
by J. Reece Roth, Glenn A. Gerdin, ${ }^{*}$ and Richard W. Richardson ${ }^{*}$ \\ Lewis Research Center
}

SUMMARY

Experimental observations have been made during steady-state operation of the NASA Lewis bumpy-torus plasma at input powers up to 150 kilowatts in deuterium and helium gas and with positive potentials applied to the midplane electrodes. In this steady-state ion heating method a modified Penning discharge is operated in a bumpytorus confinement geometry such that the plasma is acted upon by a combination of strong electric and magnetic fields. Experimental investigation of a deuterium plasma revealed electron temperatures from 14 to 140 electron volts and ion kinetic temperatures from 160 to 1785 electron volts. At least two distinct modes of operation exist, each of which is associated with a characteristic range of background pressure and electron temperature and each of which has a characteristic radial profile of electron temperature and number density. Experimental data show that the average ion residence time in the plasma is virtually independent of the magnetic field strength. Data were taken in the "standard" configuration, in which all 12 anode rings were at high voltage. Data were also taken in other symmetric configurations in which the toroidal plasma was generated by applying positive potentials to six anode rings, three anode rings, and a single anode ring, respectively.

\section{INTRODUCTION}

The plasma in the NASA Lewis bumpy-torus facility is generated by a modified Penning discharge operated in conjunction with the bumpy-torus magnetic field geometry. The resulting combination of electric and magnetic fields acting on the plasma heats the ions to high kinetic temperatures. The electric fields are applied to the plasma in such a way that they influence the stability and confinement of the plasma.

\footnotetext{
* NASA-NRC Postdoctoral Resident Research Associate.
} 
The entire experiment, including the confining magnetic field and the high positive potentials that heat the plasma, is operated in the steady state.

The bumpy-torus configuration receives its name from the shape assumed by the confined plasma. It consists of a number of coils (12 for the Lewis facility) arranged as indicated schematically in figure 1. The plasma assumes the form of a toroidal ring

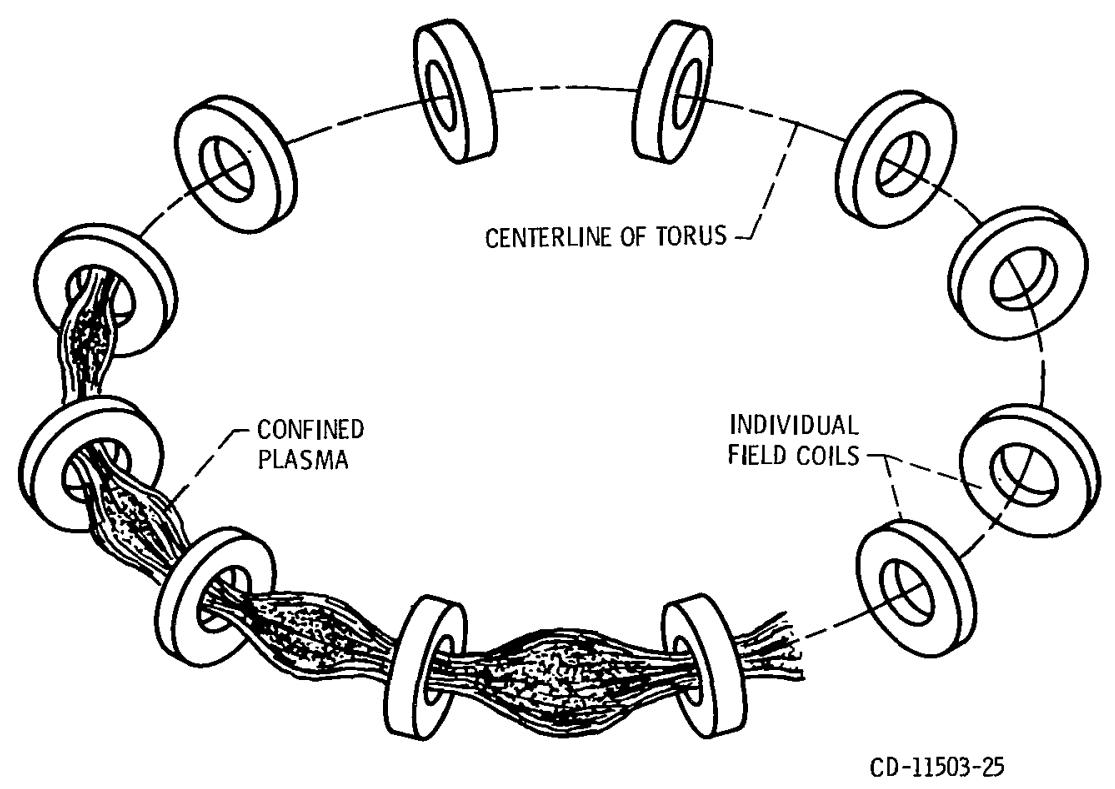

Figure 1. - Schematic drawing of bumpy-torus magnetic confinement geometry.

with bumps in the region of weak field between the coils. This confinement geometry, in the form used in the Lewis facility, was proposed by Gibson, Jordan, and Lauer (ref. 1), who later performed an extensive series of investigations of single-particle motion relevant to this geometry (refs. 2 to 5). Somewhat later, Geller (refs. 6 and 7 ) operated a pulsed plasma source in a bumpy-torus geometry and reported nearly classical confinement of the afterglow plasma (ref. 7). Fanchenko, et al. (ref. 8) have investigated turbulent heating in a bumpy-torus plasma, and recently Sprott (ref. 9) has examined the effects of asymmetries and magnetic field perturbations on particle confinement in a bumpy torus.

The bumpy-torus experiment at the NASA Lewis Research Center is part of a program of research into the problems of ion heating, high-temperature plasma physics, and thermonuclear power production associated with space applications (refs. 10 and 11). The bumpy-torus experiment was preceded by approximately 9 years of research and development on the modified Penning discharge, which was operated in a superconducting magnetic mirror facility (ref. 12). During these earlier investigations, an ion heating mechanism associated with the modified Penning discharge was experimentally 
explored and a consistent model was developed to describe the ion heating mechanism (refs. 13 to 16 ).

The general approach adopted for the NASA Lewis bumpy-torus experiment may be summarized by five major points:

(1) The plasma is confined in the bumpy-torus magnetic field configuration. The simplicity of the magnets and force-bearing structure that is possible in this configuration is advantageous in space, where mass and reliability are at a premium.

(2) The bumpy-torus experiment was designed for steady-state operation of both the magnetic field and the plasma.

(3) It was desired to advance the state of the art in superconducting magnets and other areas of technology in parallel with understanding of the basic physical processes in the plasma.

(4) Previous investigations of the modified Penning discharge (refs. 13 to 16) demonstrated that it is feasible to generate ions with a Maxwellian energy distribution and with kinetic temperatures up to several kilovolts. In the bumpy torus, the primary emphases are to understand the ion heating and confinement processes and to achieve high ion temperatures and densities and long confinement times.

(5) An additional factor that distinguishes the NASA Lewis bumpy-torus experiment from other approaches to controlled fusion that use toroidal confinement is that a high positive potential is applied to the anode rings, creating a strong radial electric field. The electric field plays an important role in the heating, confinement, and radial transport of the plasma.

This report summarizes preliminary investigations of the NASA Lewis bumpy-torus plasma that were conducted between January 1973 and November 1974. The data reported herein are restricted to the application of positive potentials to the midplane electrode rings and to information that could be extracted from a charge-exchange neutral energy analyzer, an optical spectrometer, capacitive probes, and Langmuir probes. Direct measurements of plasma density were not possible because a microwave interferometer capable of operating with this plasma was not yet available. During the experimental investigations reported herein, X-ray production, arcing, and flashover limited the applied direct-current voltage to 50 kilovolts; and heating of the surfaces in the vacuum tank limited the direct-current input power. The NASA Lewis bumpy-torus experiment cannot reach its maximum ion temperature or density until modifications and further development work remove these restrictions.

\section{DESCRIPTION OF NASA LEWIS BUMPY-TORUS FACILITY}

The characteristics and performance of the NASA Lewis bumpy-torus superconduct- 
ing magnet facility have been described elsewhere (refs. 17 and 18). The basic structure of the magnetic field in this facility is illustrated in figure 1 . The magnet array consists of 12 superconducting coils equally spaced around a toroidal volume 1.52 meters in major diameter. Each coil has a minor diameter of 19 centimeters, and the maximum designed magnetic field strength on the magnetic axis is 3.0 teslas. The minimum magnetic field on the magnetic axis between coils is 40 percent of the maximum magnetic field. The coil array is located in a single vacuum tank 2.6 meters in major diameter.

Figure 2 is an isometric cutaway drawing of the facility. Twelve airlocked access

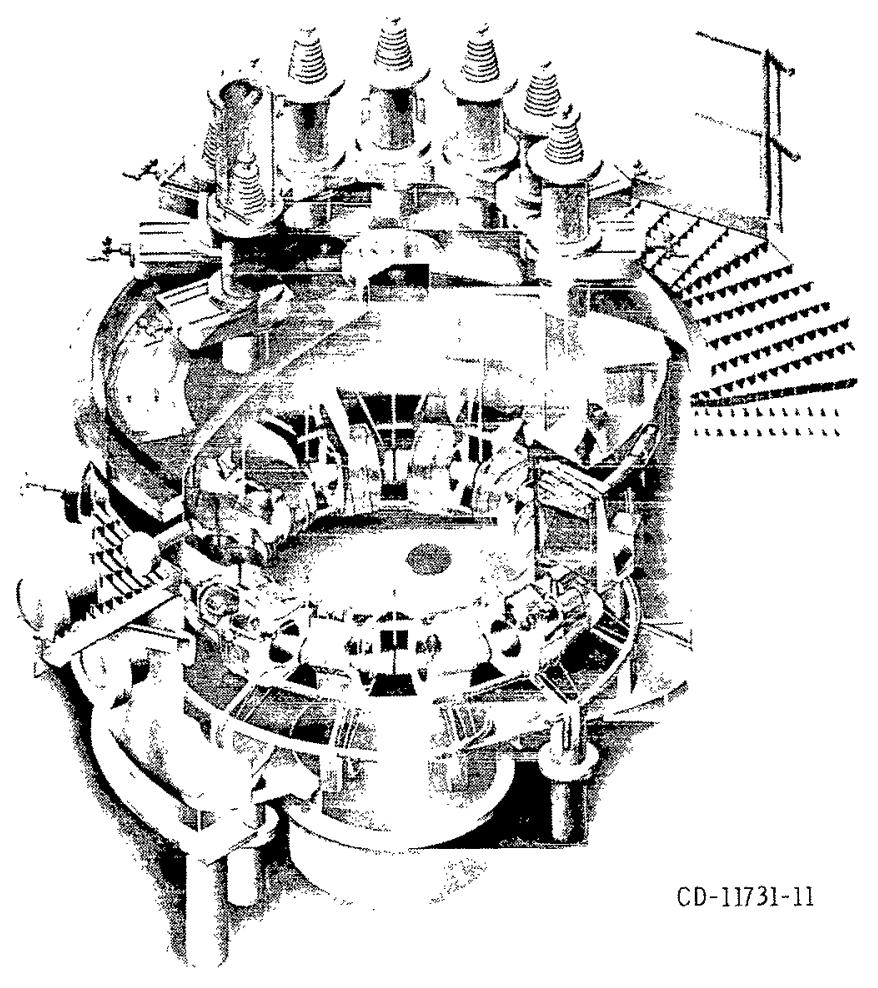

Figure 2. - Isometric cutaway drawing of NASA Lewis bumpy-torus magnet facility.

ports on the top of the vacuum tank are used to insert and withdraw the high-voltage ring electrodes into and out of the grounded coil system. Twelve 25-centimeterdiameter view ports in the equatorial plane of the torus permit visual and experimental access to the plasma volume. Twelve 16-centimeter-diameter ports are spaced on a 1. 52-meter-diameter circle on the bottom of the vacuum vessel. Some of these ports are available for instrumental access to the plasma. Figure 3 shows the bumpy-torus 
magnet facility with the top removed from the vacuum tank. Evident in this figure are the 12 superconducting coils, each with four spacer bars that support the forces exerted

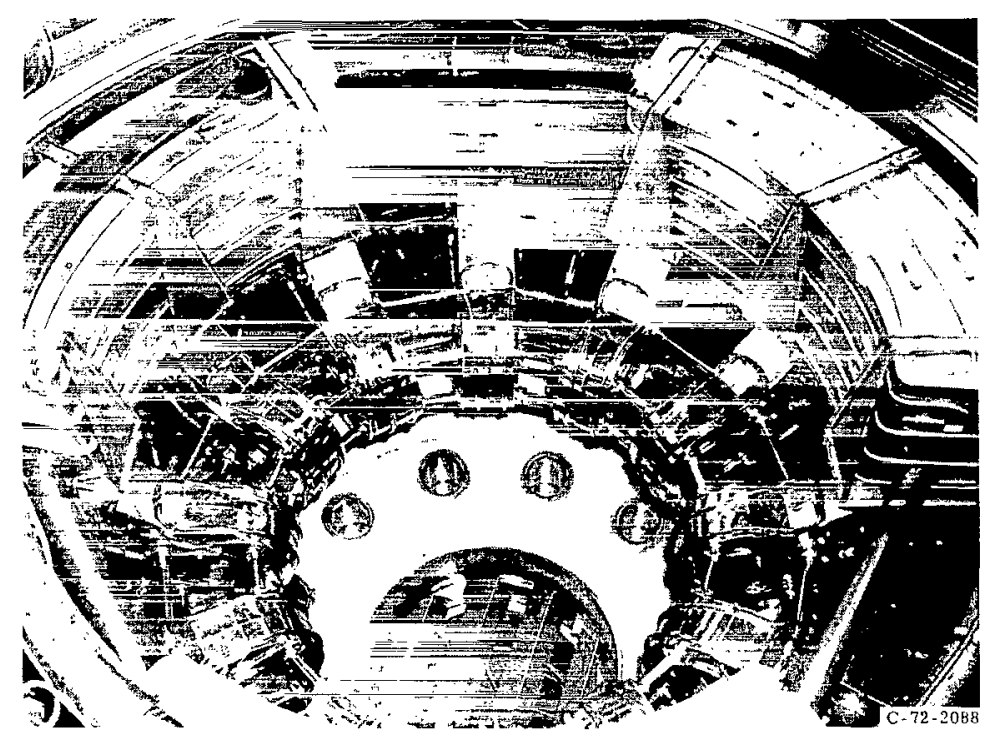

Figure 3. - NASA Lewis bumpy-torus magnet facility with vacuum tank lid removed.

by adjacent coils. At the top of the vacuum tank are three 100-liter liquid-helium reservoirs that supply the coil array. The opening of the 80-centimeter-diameter diffusion pump is visible at the bottom of the vacuum tank, as are a few of the 16-centimeterdiameter access ports. The exterior of the bumpy-torus magnet facility is shown in figure 4. At eye level are visible some of the view ports that permit visual and experimental access to the plasma volume.

The magnet facility has met and exceeded its magnetic field design goals and has been extremely reliable in its operational performance (refs. 17 and 18). Approval to construct the facility was obtained in December 1969, and the preliminary shakedown tests were completed on April 30, 1972. The final shakedown tests of the facility were completed on November 1, 1972; and the first plasma was generated in the facility on December 6, 1972. The reliability of the facility was demonstrated by a 3-month experimental program, from mid-January to mid-April 1974, in which the facility was under vacuum for the entire time. Data were taken during 45 working days.

The 1-megawatt direct-current power supply used to heat the plasma is shown in figure 5. This power supply consists of two independent modules, each capable of 50 kilovolts at 10 amperes. These modules can be connected in series to produce 100 kilovolts at 10 amperes or in parallel to produce 50 kilovolts at 20 amperes, depending on the plasma impedance at the intended operating conditions. 


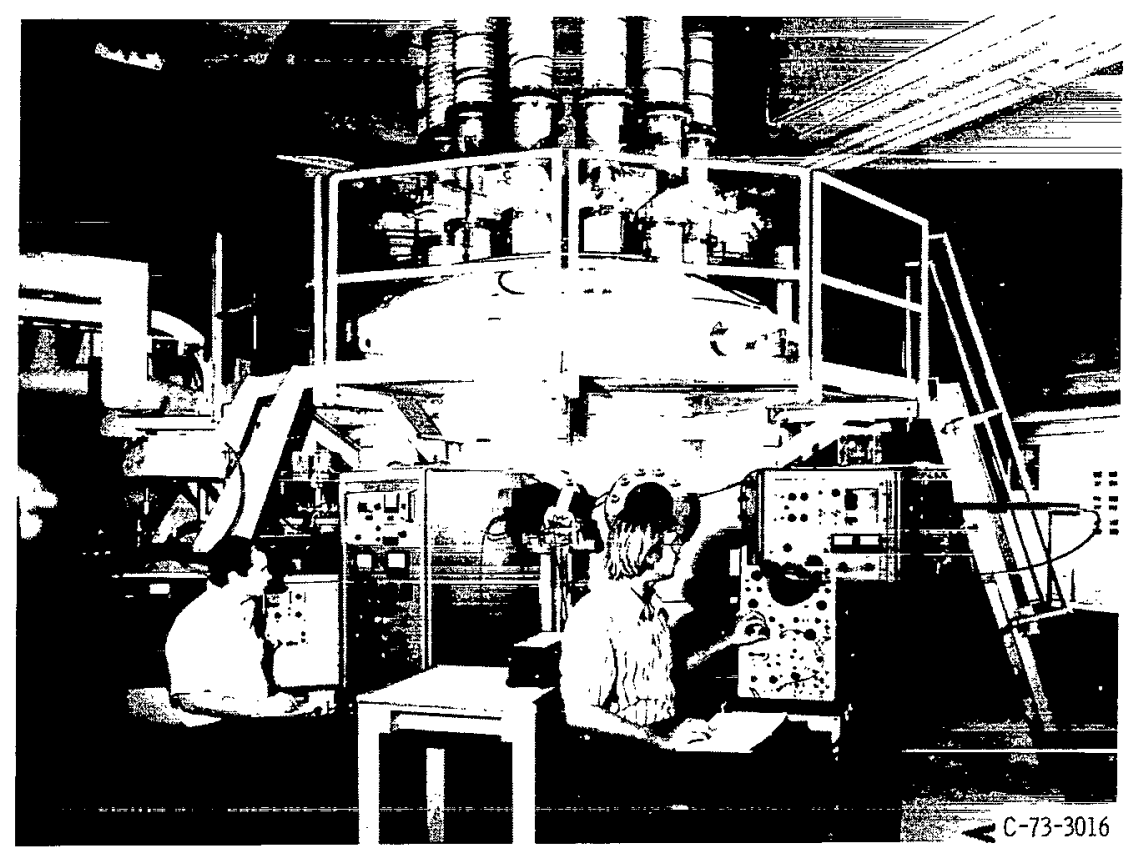

Figure 4. - Exterior of NASA Lewis bumpy-torus magnet facility, with plasma diagnostic equipment.

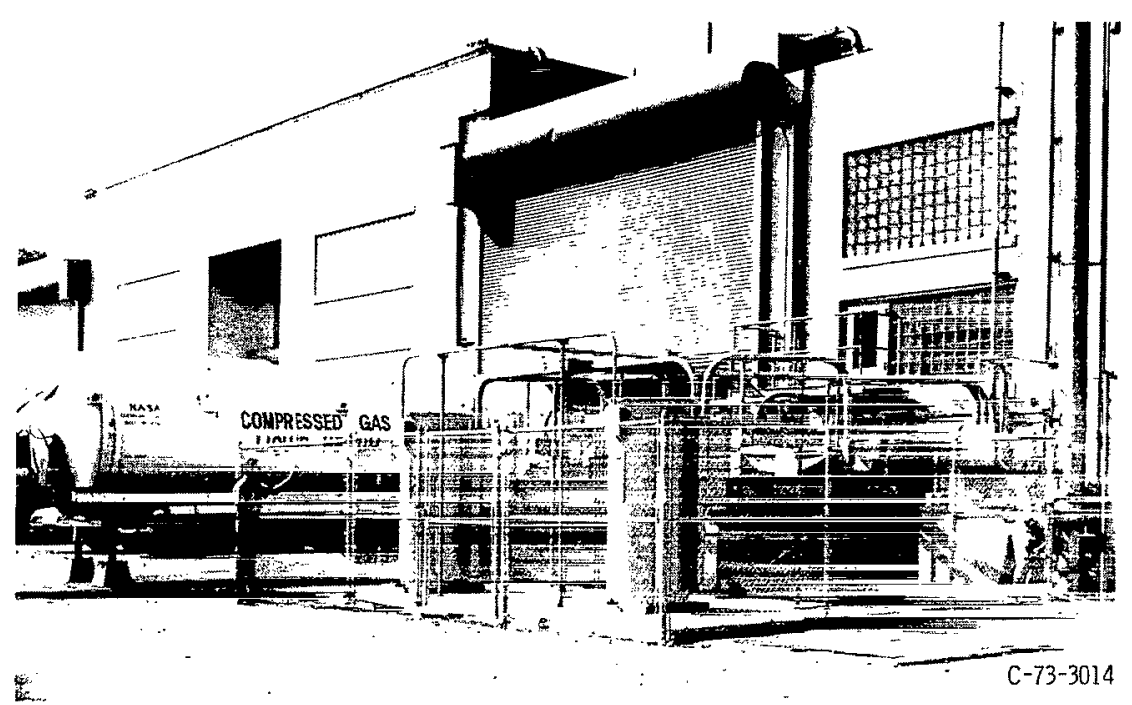

Figure 5. - High-voltage direct-current power supply used for ion heating with modified Penning discharge. (The two 50-kV, 10-A modules may be connected in series or parallel.) 


\section{CHARACTERISTICS OF BUMPY-TORUS PLASMA}

The bumpy-torus plasma is shown in figure 6, viewed through one of the 25 -centimeter-diameter glass view ports in the equatorial plane of the torus. The vertical element in the foreground is the near side of an 18-centimeter-inside-diameter water-cooled anode ring located at the midplane of the plasma. The anode ring is maintained at positive direct-current potentials up to 50 kilovolts. The plasma follows the magnetic field lines and necks down into the throats of the two adjacent magnetic field coils. Visible to the left of center in the background is the anode ring and the plasma at the opposite diameter of the toroidal array. The ions and electrons are heated by $\mathrm{E} \times \mathrm{B}$ drift in the strong crossed electric and magnetic fields that exist between the midplane electrode ring and the plasma and also between the plasma and the grounded magnet dewars and walls of the vacuum tank. The plasma tends to float at a high positive potential when a positive voltage is applied to the circular midplane electrode. Because the plasma is not completely enclosed, there is excellent visual and experimental access to it, as illustrated in figure 6. Also it may be operated with less plasmawall interaction than would exist in a completely enclosed plasma.

Some experimental runs were taken in pure helium gas to facilitate spectroscopic observations. The results of these investigations are reported in reference 19 . Other

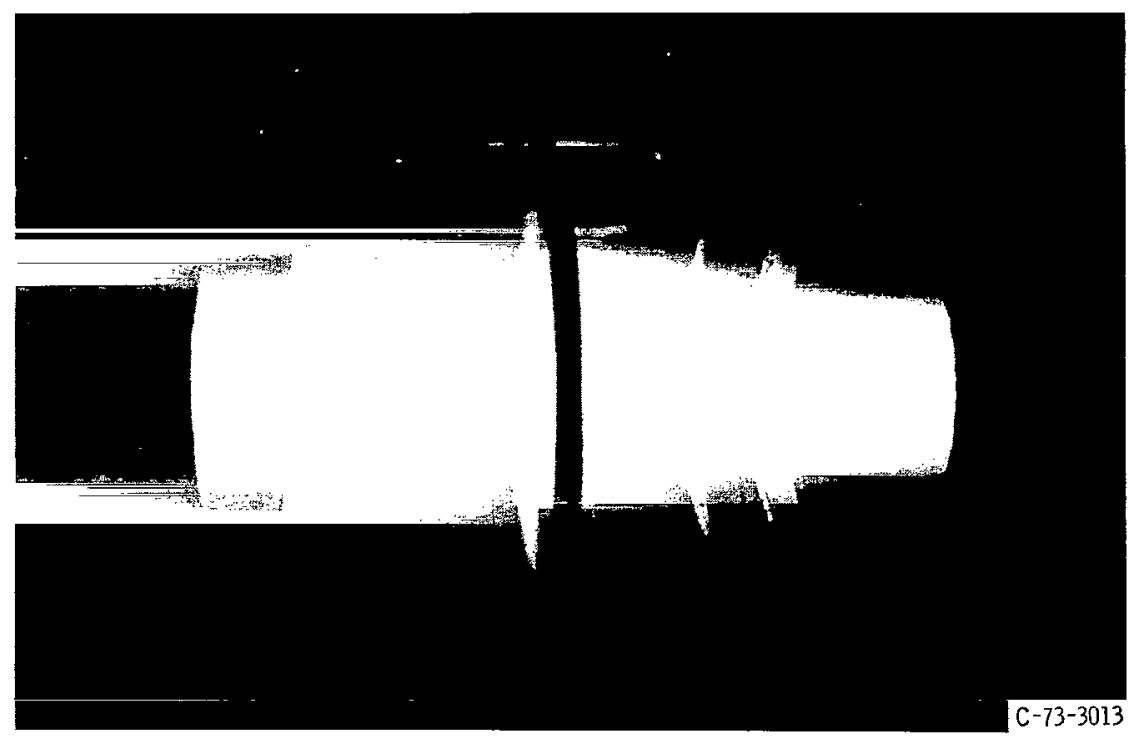

Figure 6. - Bumpy-torus plasma viewed along equatorial plane of torus. The plasma at opposite diameter of torus is visible to left of the anode ring in the foreground. I 
experimental runs were made either in pure deuterium or, when spectroscopic data were being taken, in deuterium with an admixture of helium.

Diagnostic investigations showed that the plasma characteristics were similar to those observed in the modified Penning discharge and described in references 14 to 16. The range of independent and dependent plasma parameters is indicated in table $\mathrm{I}$. The error associated with the first four plasma parameters is no more than about 5 percent; it is about 10 percent for the background neutral gas pressure. The reproducibility of the electron and ion kinetic temperatures is within 15 percent. The electron energy was typically a factor of 5 to 20 lower than the ion energy. The plasma can be operated in the steady state at power input levels up to 60 kilowatts. Power levels as high as 150 kilowatts have been maintained for periods of approximately 30 seconds. The power inputs have been limited only by heating of the uncooled sheet metal surrounding the plasma. In deuterium gas, ion kinetic temperatures have ranged from 160 to 1785 electron volts, and electron temperatures have ranged from 14 to 140 electron volts. The direct-current input power is preferentially dumped into the ion population by the Penning discharge. Estimated number densities on the axis of a helium plasma were as high as $10^{11}$ per cubic centimeter at an average ion residence time of 0.5 millisecond.

Significant features of the modified Penning discharge in the bumpy-torus geometry are that no thermionically emitting electrodes are in contact with the plasma and that no electrodes of any description are inside the plasma volume to emit secondary electrons. In addition, there are no parallel electric and magnetic fields at the electrode surfaces near the plasma. Only the water-cooled anode rings are near the plasma, and they are separated from it across the magnetic field lines in the anode sheath. Spectroscopic evidence (ref. 19) indicates that the anode currents are proportional to the particle loss rate. This further implies that the electrical circuit to the power supply is completed by ions that are transported across the magnetic field lines to the grounded walls surrounding the plasma rather than by electrons emitted from cathode surfaces. The modified Penning discharge is thus distinguished from arcs or reflex discharges, in which a heated electron-emitting cathode is in direct contact with the plasma and plays a necessary role in its operation.

An equivalent circuit of the bumpy-torus plasma is illustrated in figure 7 . The plasma is confined on magnetic field lines that close on themselves around the torus and is surrounded by a circular midplane electrode between the coils at 12 or fewer of the magnetic field midplanes. When the midplane electrode is operated as an anode and positive potentials are applied, electrons flow from the plasma to the anode ring and ions flow from the plasma to the grounded magnet dewars. The anode sheath between the anode ring and the plasma has an effective resistance $R_{a}$, and the cathode sheath between the plasma and the grounded dewars has an equivalent resistance of $R_{c}$. The potential assumed by the plasma depends on the relative value of these two equivalent 
TABLE I. - RANGE OF EXPERIMENTAL PARAMETERS ${ }^{\mathrm{a}}$

\begin{tabular}{|c|c|c|c|c|}
\hline \multirow[t]{2}{*}{ Parameter } & \multicolumn{2}{|c|}{ Helium } & \multicolumn{2}{|c|}{ Deuterium } \\
\hline & Low value & High value & Low value & High value \\
\hline Anode voltage, $\mathrm{V}_{\mathrm{a}}, \mathrm{kV}$ & 1 & 50 & 1 & 50 \\
\hline Anode current, $\mathrm{I}_{2}, \mathrm{~A}$ & 0.001 & 5.5 & 0.001 & 5.3 \\
\hline Input power, $\mathrm{w}_{\mathrm{p}}, \mathrm{w}$ & 0.15 & $10^{5}$ & 0.03 & 1. $5 \times 10^{5}$ \\
\hline Maximum magnetic field strength, $B_{\max }, T$ & 0.24 & 2.7 & 0.24 & 3.0 \\
\hline Background neutral gas pressure, $p_{t}, P_{a}$ (torr) & $7.3 \times 10^{-4}\left(5.5 \times 10^{-6}\right)$ & $2.5 \times 10^{-2}\left(1.9 \times 10^{-4}\right)$ & $4.9 \times 10^{-4}\left(3.7 \times 10^{-6}\right)$ & 1. $8 \times 10^{-2}\left(1.32 \times 10^{-4}\right)$ \\
\hline Electron temperature, $\mathrm{T}_{\mathrm{e}}, \mathrm{eV}$ & 11 & 95 & 14 & 140 \\
\hline Ion kinetic temperature, $\mathrm{T}_{\mathfrak{j}}, \mathrm{eV}$ & 650 & 3300 & 160 & 1785 \\
\hline Average number density on axis, $\mathrm{n}_{\mathrm{e}}(0)$, per $\mathrm{cm}^{3}$ & $\sim 10^{8}$ & $10^{11}$ & - & - \\
\hline
\end{tabular}

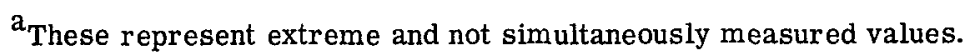




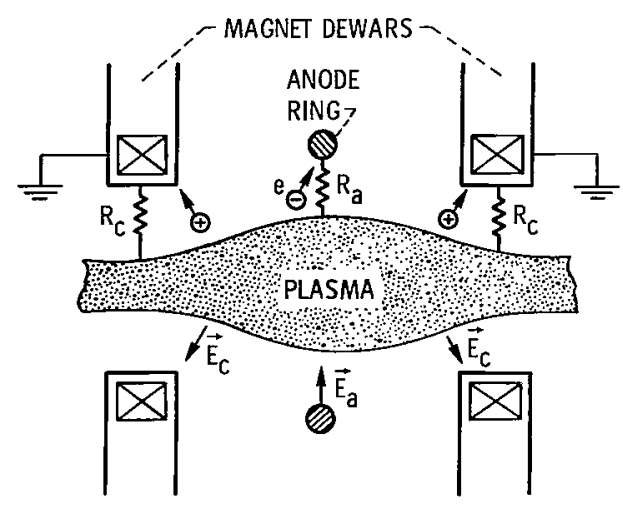

Figure 7. - Equivalent circuit of bumpy-torus plasma.

resistances. This value is determined in turn by the relative mobility of ions and electrons across the cathode and anode sheaths, respectively, as well as by the sheath geometry and other plasma properties.

It was originally anticipated that the ions would be the most mobile species in the strong magnetic fields applied and that $R_{c}$ would be considerably less than $R_{a}$. In fact, this proved not to be the case. The processes that occur in the two sheaths are such that the value of $R_{c}$ is significantly greater than the value of the anode sheath resistance. As a result, the plasma tends to assume a positive potential close to that of the circular anode rings in the midplane. With the midplane electrode operated as an anode, the electric fields point radially inward in the anode sheath and radially outward between the plasma and the grounded superconducting magnet dewars.

\section{OPERATING REGIMES OF BUMPY-TORUS PLASMA}

The current-voltage characteristics of the bumpy-torus plasma are shown in figure 8 (a) for deuterium gas and in figure $8(\mathrm{~b})$ for helium gas. The plasma is characterized by at least three distinct regimes of operation. The so-called high-pressure mode is apparent at the higher neutral gas pressures represented by the curves in the upper left of figures 8(a) and (b). In the high-pressure mode with all 12 anode rings operating, the current is approximately proportional to the cube of the anode voltage until a critical current and voltage are reached at which the discharge spontaneously changes mode.

The low-pressure mode is represented by the curves in the lower right of figures 8(a) and (b). The low-pressure-mode data are further separated by a change of slope at approximately 10 kilovolts; the anode current is approximately proportional to the square of the anode voltage below the knee and directly proportional to anode voltage 


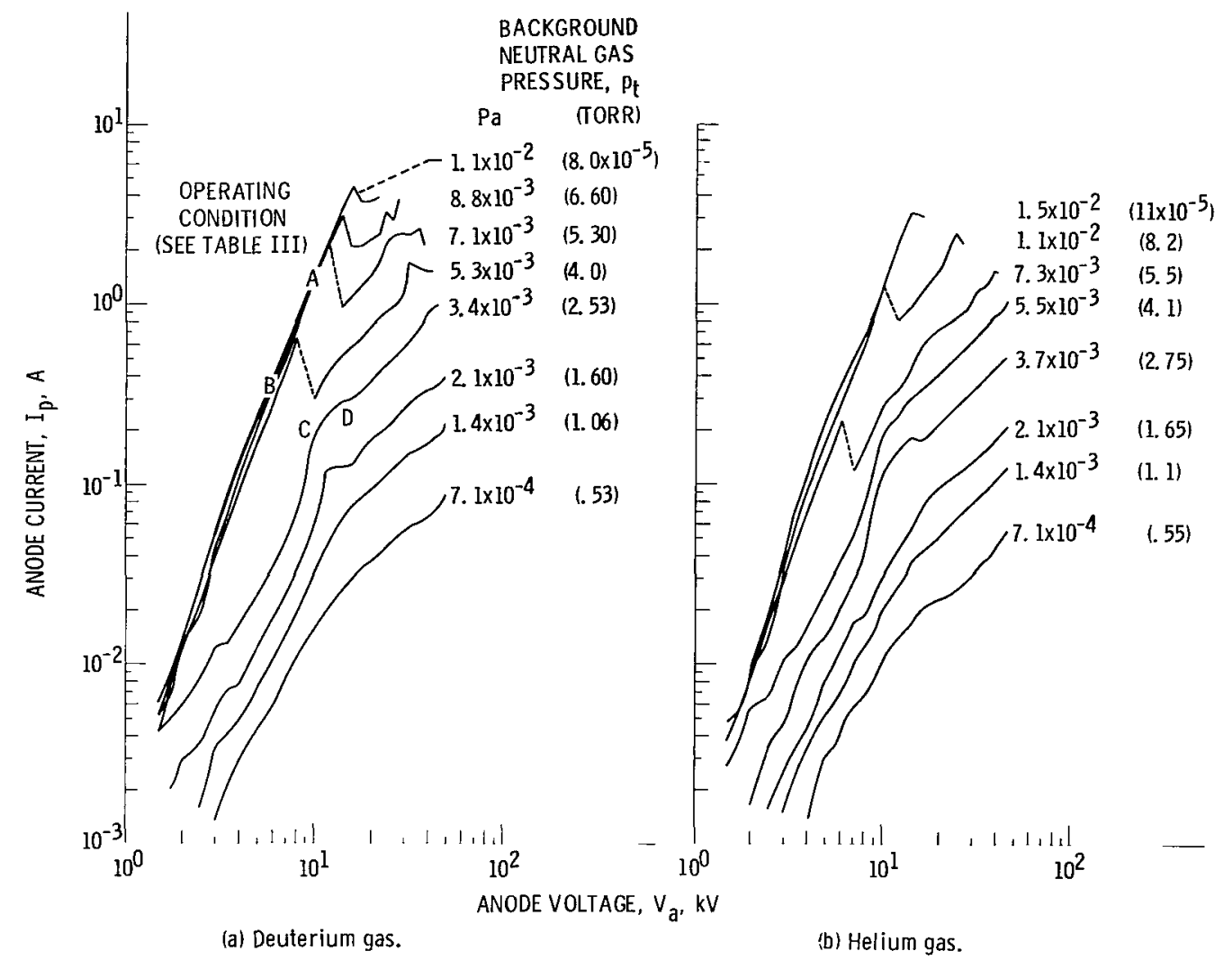

Figure 8. - Current-voltage curves of bumpy-torus plasma for eight background neutral gas pressures. Maximum magnetic field strength, $B_{\max }, 2.4$ teslas; number of anode rings, 12.

above the knee. This knee in the low-pressure-mode data appears to be associated with a transition from an $m=-1$ to an $m=-2$ minor azimuthal rotational mode number in the ion spoke rotation (ref. 20). The location of the characteristic curves on the current-voltage diagram depends upon background neutral gas pressure. The sensitivity of anode current to neutral pressure at a fixed anode voltage is the basis for using the Penning discharge as a vacuum gage (ref. 21). These current-voltage curves are continuous except at intermediate pressures, where a sharp discontinuity (indicated by dashed lines in fig. 8) exists at the mode transition point.

An attempt was made to determine the physical processes and parameters that characterize the operating regimes of the bumpy-torus plasma. The behavior of the current-voltage curves and of the mode transitions in figure 8 are qualitatively the same for deuterium and helium gas and exhibit only relatively small quantitative differences. Both gases display a high- and a low-pressure mode, and the current-voltage curves have the same characteristic slopes in analogous regions of the current-voltage diagram. The similarity of the current-voltage curves for these two gases suggests that the high- 
and low-pressure modes of operation do not occur because of metastable production or as a result of processes that depend on either a diatomic or monatomic molecular structure.

It was thought that charge-exchange processes might play a role in determining the operating regime of the plasma. Figure 9(a) shows experimentally measured ion temperature as a function of electron temperature in deuterium gas. This plane is divided by two curved lines, which are obtained by setting the charge-exchange times of $\mathrm{D}^{+}$on $\mathrm{D}_{2}$ and of $\mathrm{D}_{2}^{+}$on $\mathrm{D}_{2}$ equal to the ion residence time in the plasma. It is shown in the section on particle transport that the ion residence time is equal to the ionization time in this steady-state plasma. The ionization time can be calculated from the spectroscopically determined electron temperature and the background neutral gas pressure. In deuterium gas, all data were taken in the region in which the charge-exchange time was longer than the average ion residence time. The deuterium ions have a higher probability of being lost from the plasma than they have of undergoing a charge exchange. Figure 9(b) shows similar data plotted for helium gas. The helium data lie in the region in which the average ion residence time is longer than the charge-exchange time. In helium gas, a helium ion will charge exchange, on the average, long before its charge is lost to the walls. The fact that charge-exchange processes dominate in helium, while in deuterium they do not, tends to rule out charge exchange as a cause of the similar high- and low-pressure-mode behavior observed in the two plasmas.

The mode of operation of the discharge appeared to be characterized by only two pa-

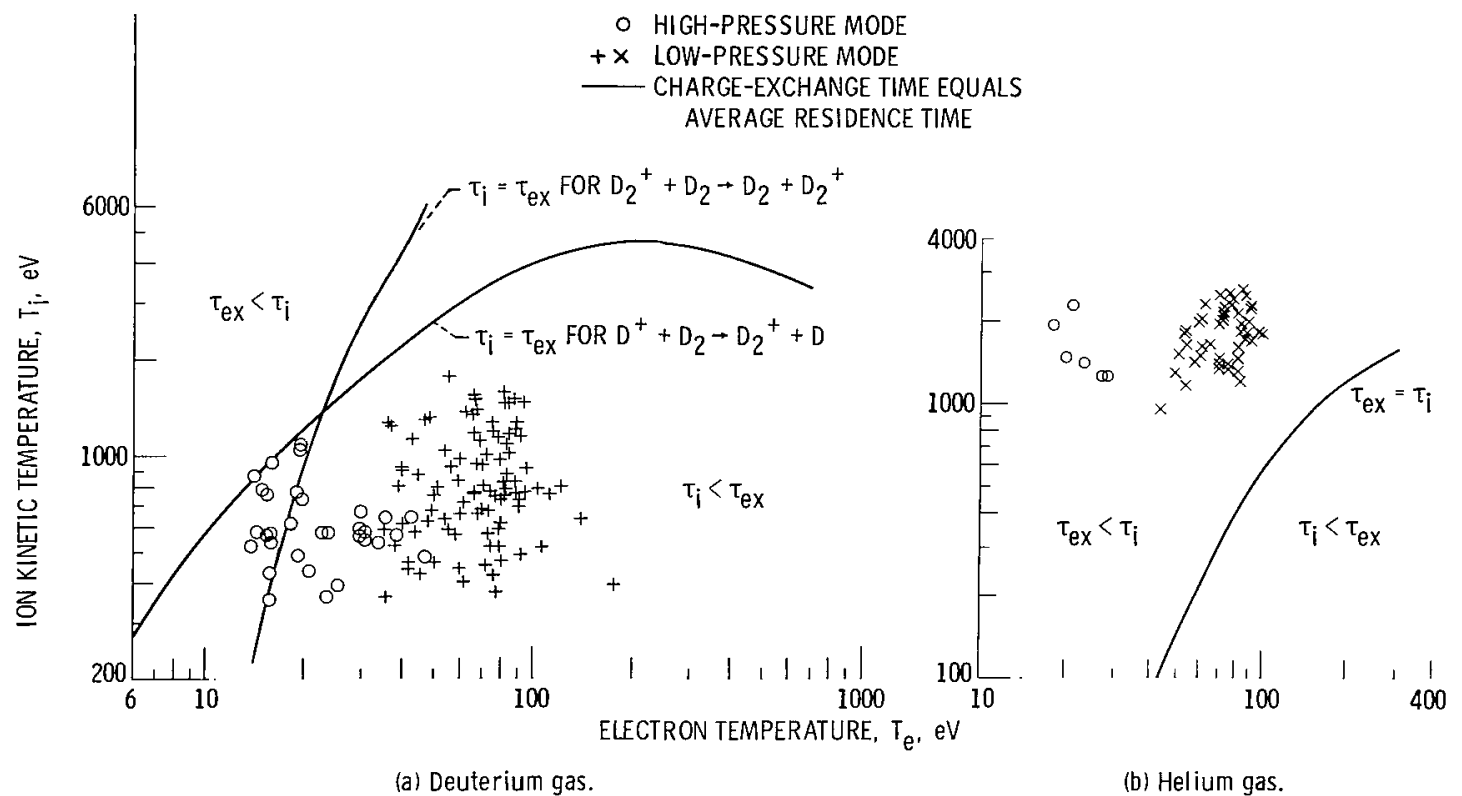

Figure 9. - Ion kinetic temperature as function of electron temperature for bumpy-torus plasma. Maximum magnetic field strength, $B_{\max }, 2.4$ teslas. 
rameters, the neutral number density and the electron temperature. Figures 10(a) and (b) give the background neutral gas pressure and the spectroscopically determined electron temperature for the two modes of operation in deuterium and helium gas, respectively. For both gases, there is a minimum pressure below which the highpressure mode is not observed. This minimum pressure is approximately $3.6 \times 10^{-3}$ pascal (2. $7 \times 10^{-5}$ tor $\left.r\right)$ in deuterium gas and $6.7 \times 10^{-3}$ pascal $\left(5 \times 10^{-5}\right.$ tor $\left.r\right)$ in helium gas. In addition, a critical electron temperature of about 35 electron volts separates the two modes of operation in both gases. The boundaries of the operating regimes indicated in figure 10 do not bear any obvious relation to the mean free paths or particle residence times in these plasmas, and their physical significance is as yet unknown.

Spectroscopic investigations showed that the radial profiles of relative number density, electron temperature, and ionization rate differed in a characteristic manner in the two modes of operation. The physical process responsible for triggering the mode transition is not understood at present and is a subject of continuing investigation.

Figures 11(a) and (b) show the direct-current plasma $\left(R_{p}=V_{a} / I_{p}\right)$ impedance as a function of plasma current and background neutral gas pressure for deuterium and helium gas, respectively. The resistance tends to increase as the pressure is lowered. The data for the high-pressure mode tend to fall along a simple power-law correlation. The plasma input power is shown as a function of plasma current in figure 12(a) for deuterium gas and in figure 12(b) for helium gas. The data tend to follow a simple powerlaw correlation, which in the high-pressure mode covers over six orders of magnitude in the input power. That no departure from these correlations is observed at the high end indicates that the same physical processes probably determine the plasma impedance over this entire range.

The data in figure 12 suggest a plasma power-law correlation of the form

$$
\mathrm{w}_{\mathrm{po}}=\frac{\mathrm{r}_{\mathrm{p}}^{\mathrm{A}}}{\mathrm{p}_{\mathrm{t}}^{\mathrm{B}}}
$$

where $I_{p}$ is the anode current flowing to the plasma and $p_{t}$ is the neutral gas pressure in the vacuum tank. The exponents $\mathrm{A}$ and $\mathrm{B}$ were adjusted to obtain a best fit to the experimental current-voltage curves. This was done with a logarithmic least-squares computer program, and the resulting best-fitting exponents are listed in table II. Separate exponents were calculated for deuterium and helium plasmas and for the low - and high-pressure modes of operation. All four sets of data include a factor of 10 variation in magnetic field. This is why the exponents in table $\Pi$ differ slightly from those derived from the slopes of the current-voltage curves in figure 8, which were taken at a single magnetic field. The exponents of this power-law correlation are in fair agree- 


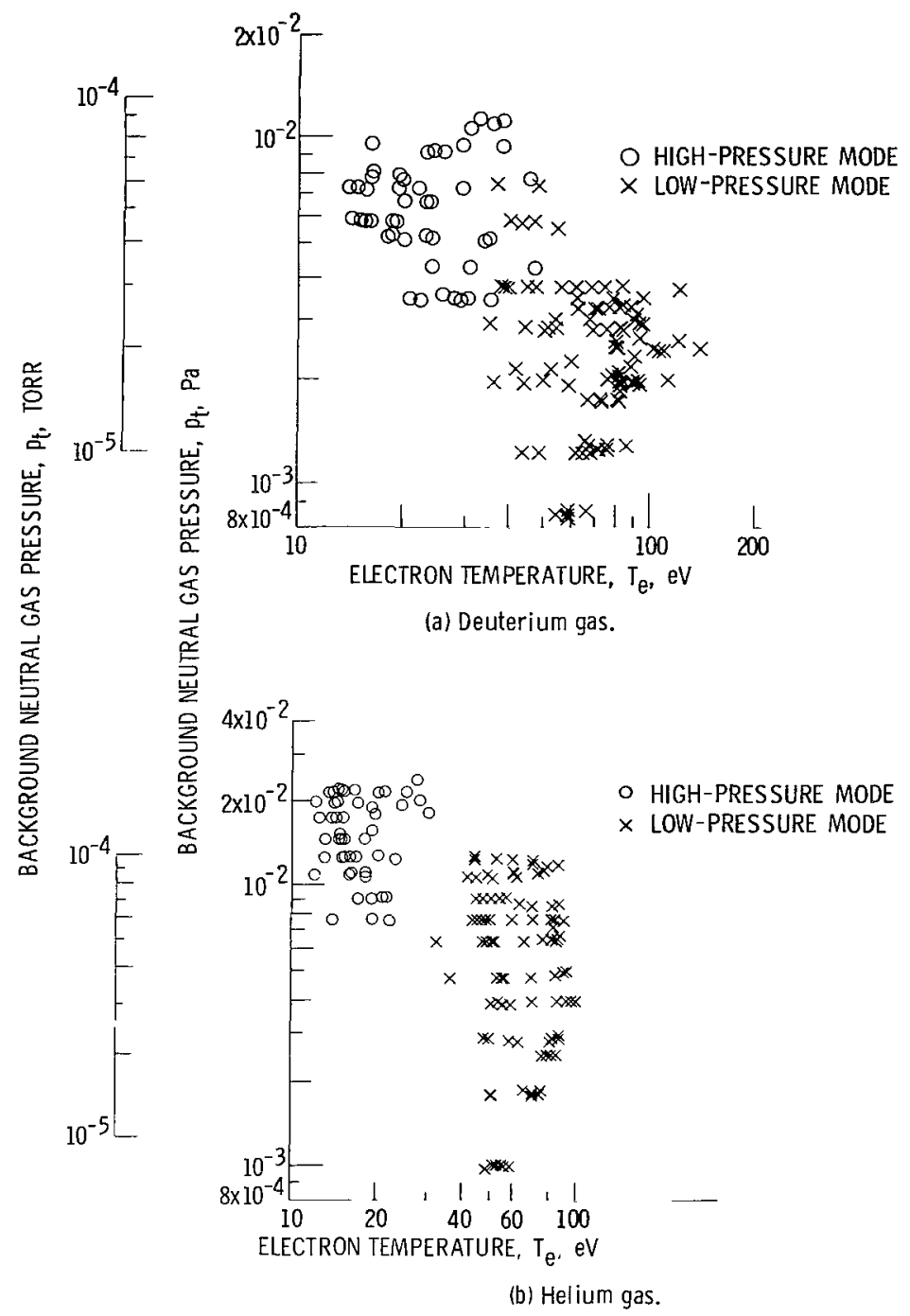

Figure 10. - Operating regimes of high- and low-pressure modes in background neutral gas pressure - electron temperature plane. Maximum magnetic field strength, $B_{\max }, 2.4$ teslas. 


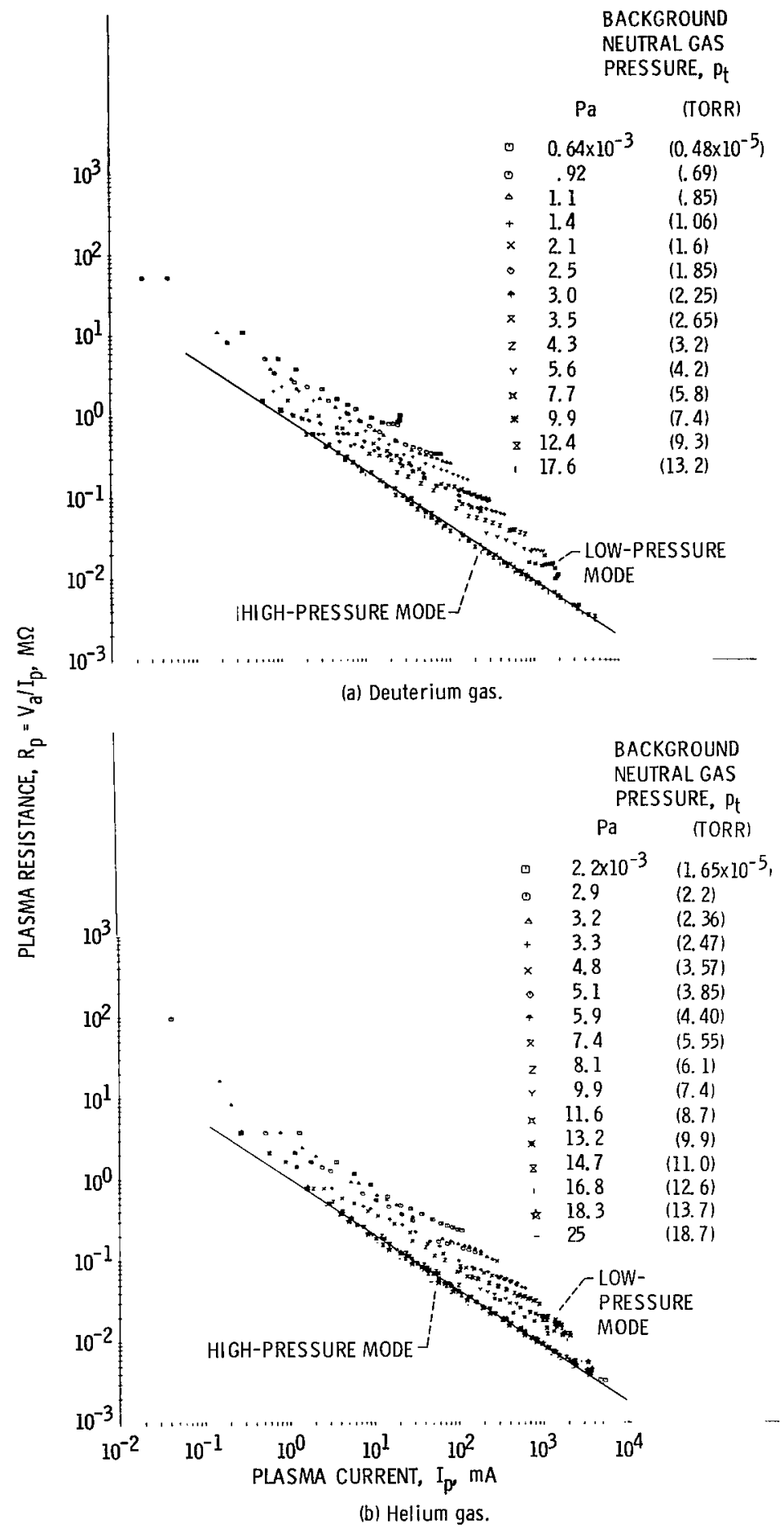

Figure 11. - Direct-current plasma resistance as function of anode current flowing to plasma at various background neutral gas pressures. Maximum magnetic field strength, $B_{\max }, 2.4$ teslas. 


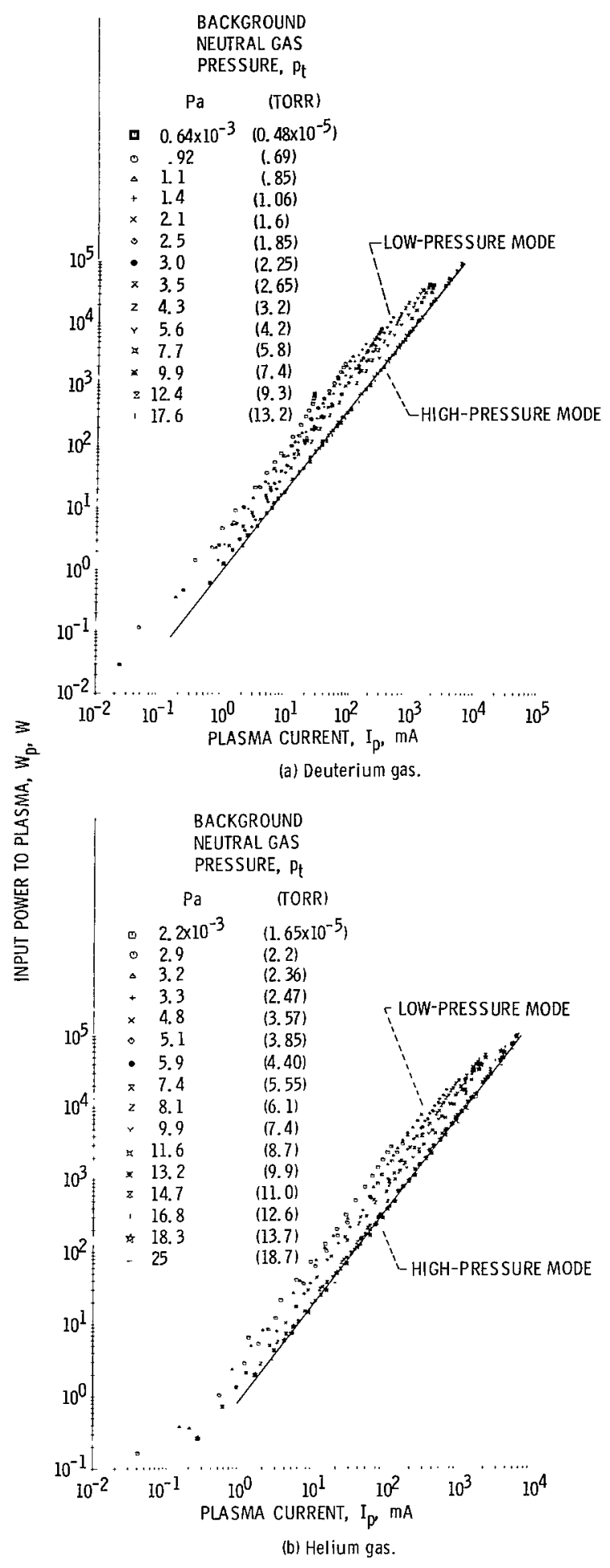

Figure 12. - Input power to plasma as function of anode current flowing to plasma at various background neutral gas pressures. Maximum magnetic field strength, $B_{\max }, 2.4$ teslas. 

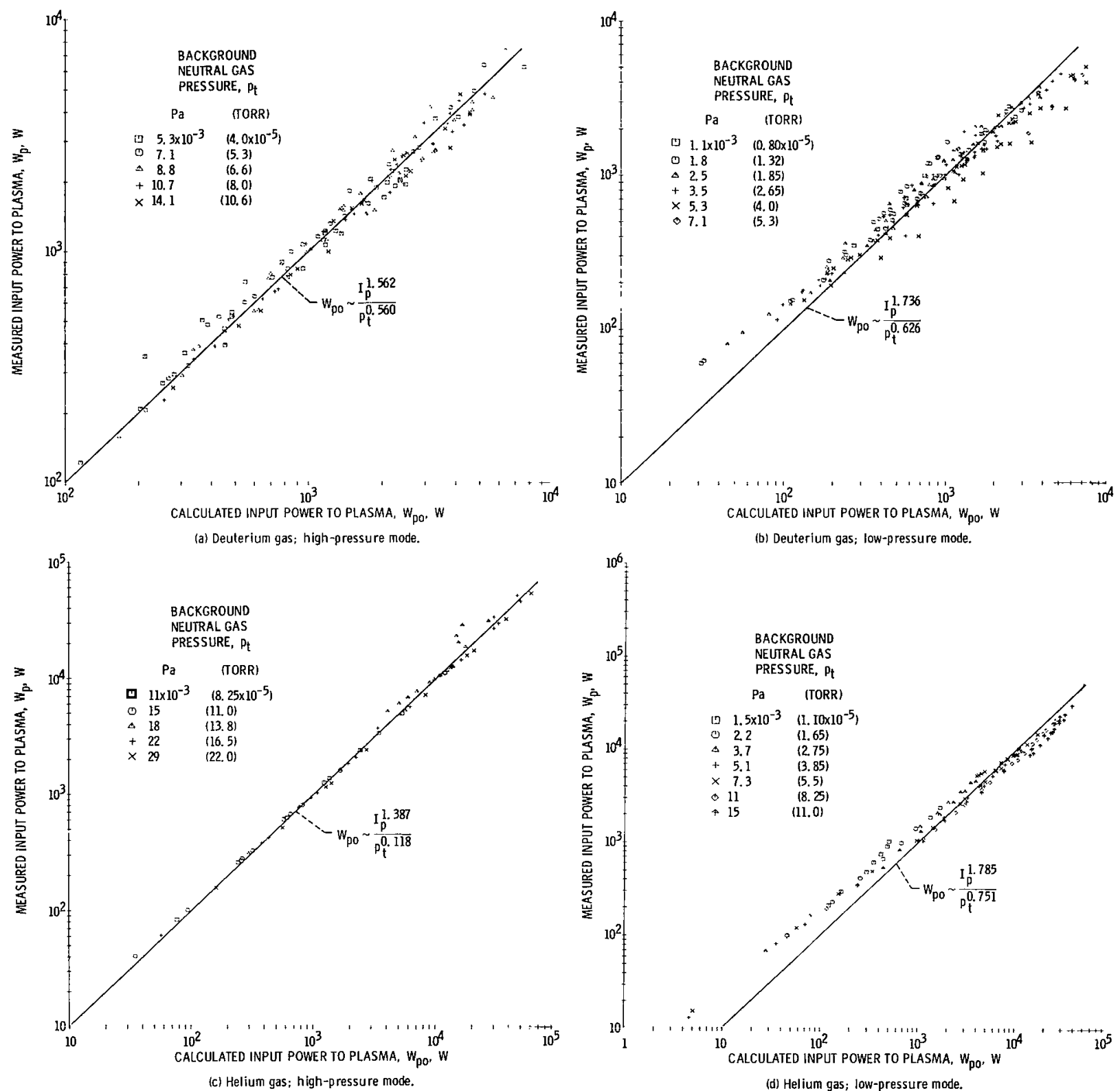

Figure 13. - Measured input power to bumpy-torus plasma as function of calculated input power (eq. (1)) for best-fitting parameter exponents (indicated in each graph). 
TABLE II. - BEST-FITTING PARAMETERS

FOR PLASMA POWER CORRELATION

\begin{tabular}{|c|c|c|c|}
\hline \multirow[t]{2}{*}{ Gas species } & \multirow{2}{*}{$\begin{array}{c}\text { Mode of oper- } \\
\text { ation }\end{array}$} & \multicolumn{2}{|c|}{ Exponent } \\
\hline & & A & B \\
\hline \multirow[t]{2}{*}{ Deuterium } & Low pressure & 1. 736 & 0.626 \\
\hline & High pressure & 1. 562 & .560 \\
\hline \multirow[t]{2}{*}{ Helium } & Low pressure & 1. 785 & .751 \\
\hline & High pressure & 1.387 & a. 118 \\
\hline
\end{tabular}

ment for both gases, except for the helium high-pressure-mode value, for which the range of pressure variation was inadequate.

The degree to which the exponents in table II yield a good fit may be judged by plotting the value of $W_{\text {po }}$ from equation (1) against the actual plasma input power. This has been done for both gases and both modes of operation in figure 13. In general, correlation of equation (1) with the exponents of table II yields a good description of the plasma behavior over at least two orders of magnitude. As can be seen in figure 13 the power-law correlation in the low-pressure mode is not quite as good as that in the highpressure mode. At the present stage of our understanding of the bumpy-torus plasma, equation (1) must be regarded as purely phenomenological and without theoretical justificiation. However, equation (1) summarizes a large body of experimental data and provides a point of reference for a theory of the bumpy-torus plasma.

\section{ION HEATING IN THE BUMPY TORUS}

The ion heating process in the modified Penning discharge has been extensively investigated in an axisymmetric magnetic mirror geometry (refs. 14 and 15). It was found that ion heating and thermalization are directly correlated with the $(\overrightarrow{\mathrm{E}} \times \overrightarrow{\mathrm{B}}) / \mathrm{B}^{2}$ drift of ions in the plasma sheath. The physical processes observed in these investigations are summarized schematically in figure 14. The outer circle represents the anode ring, which in these experiments is maintained at positive potentials up to 50 kilovolts. The high anode potentials give rise to a very strong electric field that points radially inward in the anode sheath and radially outward in regions outside the anode sheath. The ions are expected to be the more mobile species in directions perpendicular to the mag- 


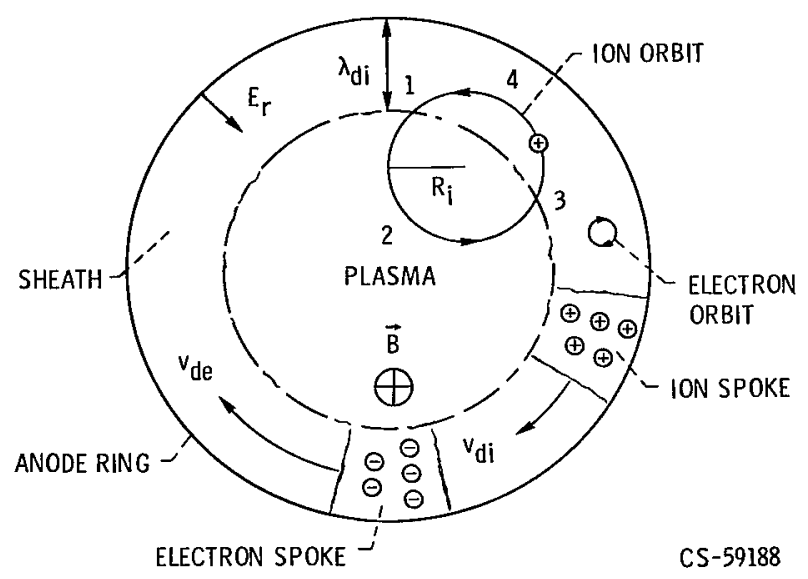

Figure 14. - Schematic illustration of physical processes in anode sheath of modified Penning discharge.

netic field, and the anode sheath thickness would then be determined by the ion Debye length $\lambda_{\mathrm{di}}$ (refs. 14 and 15). The gyrodiameter of the electrons is much smaller than the ion Debye length, so the electrons see the full electric field of the sheath and drift with the full $(\overrightarrow{\mathrm{E}} \times \overrightarrow{\mathrm{B}}) / \mathrm{B}^{2}$ drift velocity. In the modified Penning discharge, however, the ion gyro diameter is larger than the ion Debye length. The average ion spends only a fraction of its time in the electric field of the sheath and therefore sees an effective electric field much smaller than the sheath electric field. For this reason, the ions drift with an azimuthal velocity much smaller than that of the electrons, usually from $1 / 5$ to $1 / 20$ of the electron drift velocity.

Experimental evidence for spokes in the bumpy-torus plasma is shown in figure 15 (see also ref. 22). These three single-sweep oscilloscope traces show the electrostatic potential detected by a capacitive probe at three sweep speeds. The top trace shows the electron spoke trace with downward-pointing cusps as the negative charges swept by the probe with a frequency of 1.8 megahertz. The intermediate trace shows both the electron spoke trace with its downward-pointing cusps at 1.8 megahertz and the ion spoke modulation with its upward-pointing cusp at $95 \mathrm{kilohertz.} \mathrm{The} \mathrm{ion} \mathrm{spoke} \mathrm{modulation}$ is most apparent in the bottom trace. Figure 16 shows electrostatic potential waveforms from two capacitive probes at opposite ends of a minor diameter. Both the ion and electron spokes are $180^{\circ}$ out of phase and are single $\mathrm{m}=-1$ spokes in this case.

In reference 14 it was shown that the ion spoke rotational velocity in a modified Penning discharge was equal to the velocity corresponding to the most probable energy of the ions. The ion heating is therefore correlated with $(\overrightarrow{\mathrm{E}} \times \overrightarrow{\mathrm{B}}) / \mathrm{B}^{2} \mathrm{drift}$ in the plasma sheath. The ion spoke rotational velocity in the bumpy-torus plasma has also been shown to be proportional to the square root of the ion kinetic temperature. This sug- 


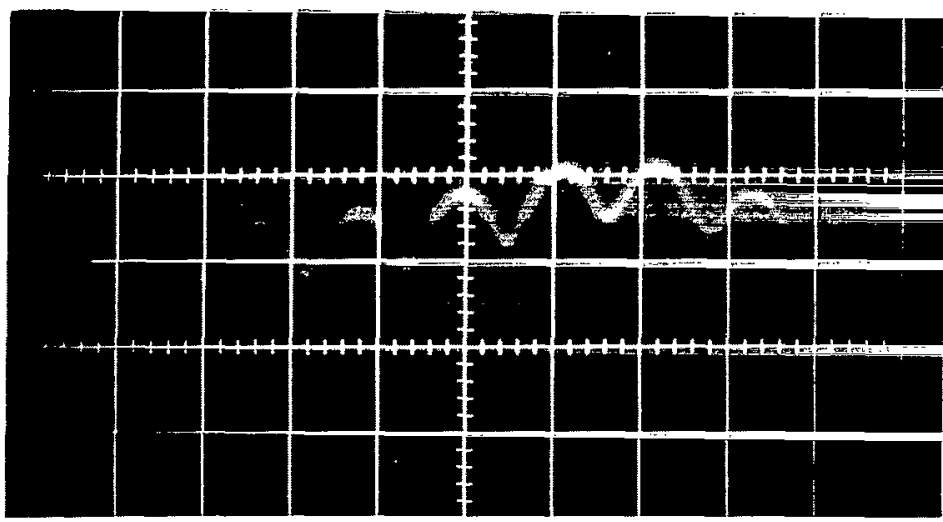

(a) Electron spoke; sweep speed, 0.5 microsecond per centimeter; frequency, $\approx 1.8$ megahertz.

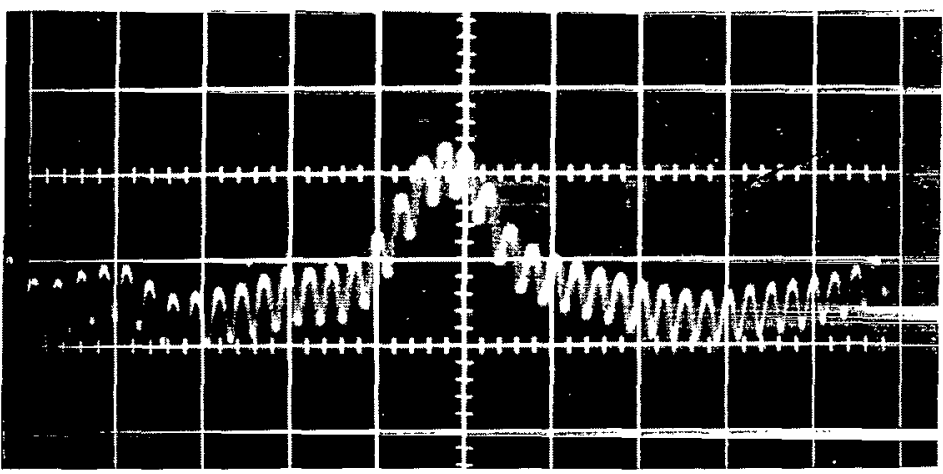

(b) Electron and ion spoke modulation; sweep speed, 2.0 microseconds per centimeter; frequency, 1.8 megahertz and 95 kilohertz.

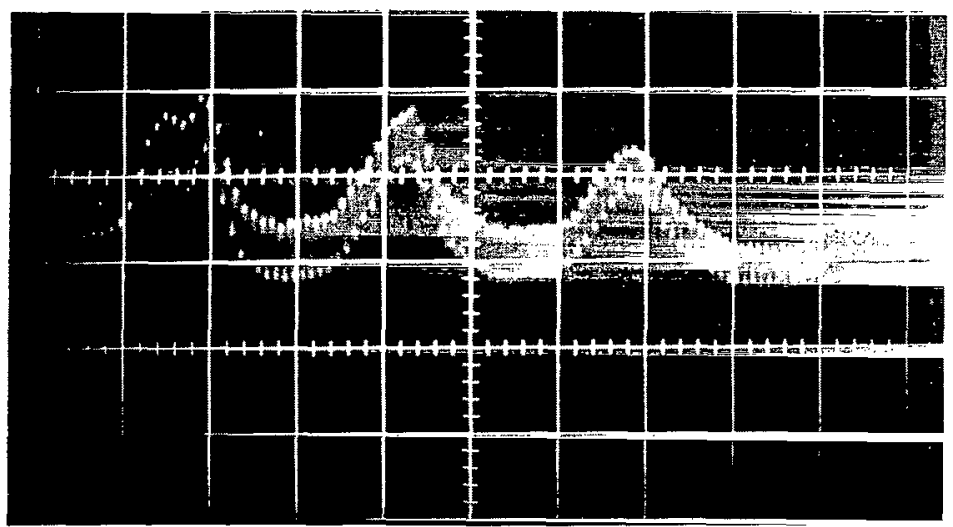

(c) Ion spoke; sweep speed, 5.0 microseconds per centimeter; frequency, $\approx 95$ kilohertz.

Figure 15. - Electrostatic potential waveform from a single capacitive probe. Positive potential gives upward deflection; anode voltage, $V_{a}, 18$ kilovolts; anode current, Ip, 76 milliamperes; background neutral gas (deuterium) pressure, $p_{t}, 7.1 \times 10^{-4}$ pascal $\left(5.3 \times 10^{-6}\right.$ torr); maximum magnetic field strength, $B_{\max }, 1.44$ teslas. 


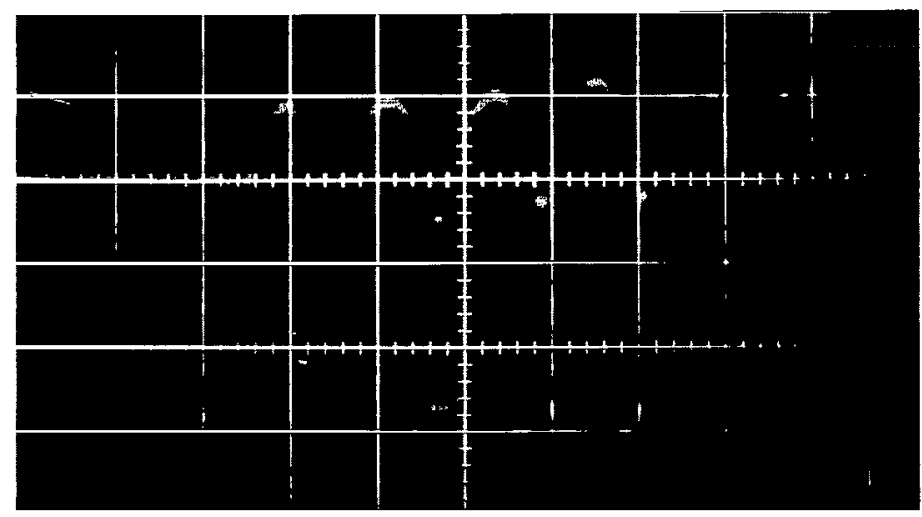

(a) Electron spokes; sweep speed, 0.5 microsecond per centimeter.

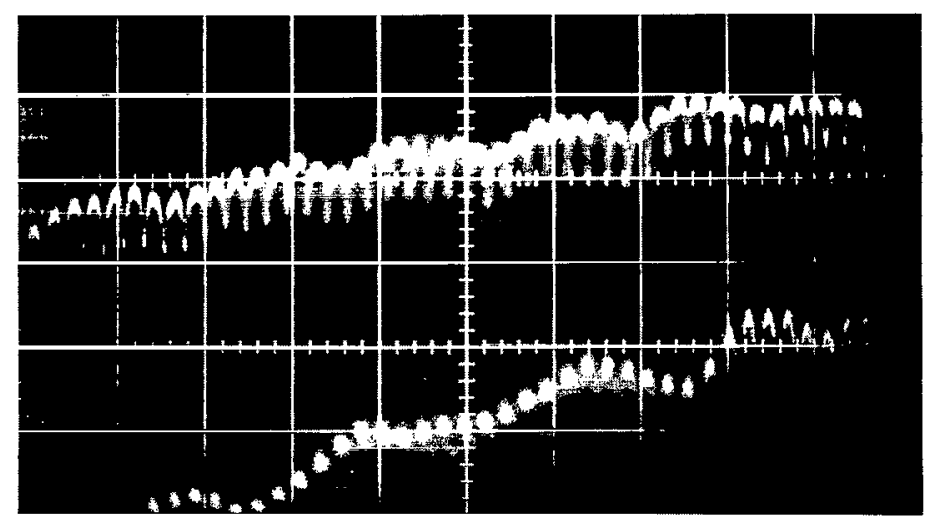

(b) Ion and electron spokes; sweep speed, 2.0 microseconds per centimeter.

Figure 16. - Electrostatic potential waveforms from two capacitive probes at opposite ends of a diameter. Positive potential gives upward deflection; operating conditions same as in figure 15.

gests that the same ion heating mechanism operates in Penning discharges in both axisymmetric magnetic mirror and bumpy-torus geometries (ref. 22). Maxwellianization of the ion energy distribution function, reported in references 13, 16, and 22, apparently results from electrostatic turbulence in which the energy fed into the turbulent spectrum at the ion spoke rotational frequency cascades upward in frequency and downward in scale size until it is finally dissipated in thermal motions of the ions.

A charge-exchange neutral particle analyzer was used to measure the energy distribution function and kinetic temperature of the ions perpendicular to the toroidal magnetic field in the bumpy torus. The analyzer is a duplicate of that developed by Valckx at Fontenay-aux-Roses (ref. 23), and the data were taken and reduced in the manner described in reference 16 . Figure 17 shows the experimental arrangement of the charge- 


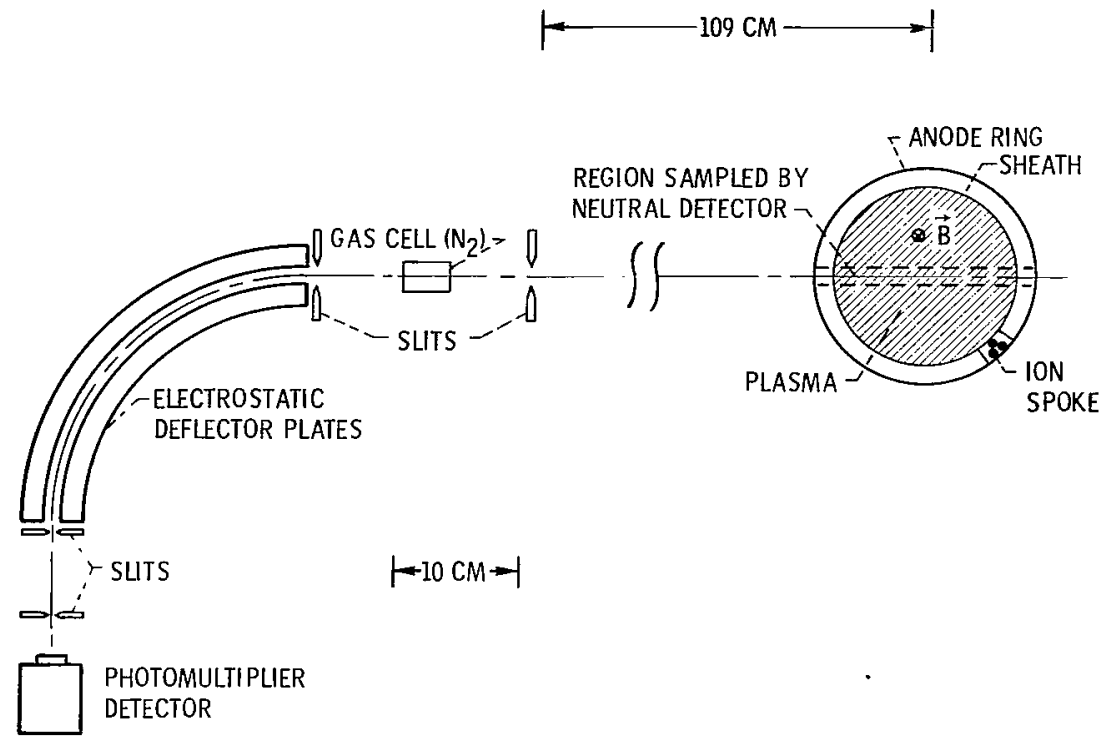

Figure 17. - Schematic drawing of charge-exchange neutral particle analyzer. (Line of sight of detector is in equatorial plane and perpendicular to axis of torus. )

exchange neutral particle analyzer with respect to the bumpy-torus plasma. The analyzer was aimed directly across the minor diameter of the plasma in the magnetic field midplane between two coils. Charge-exchange neutrals from the opposite diameter of the toroidal plasma were prevented from entering the analyzer by a baffle plate on the central axis of the torus.

Plasma ions which charge exchange with the background molecular neutral gas are sampled by the analyzer. The long mean free path of the energetic neutral deuterium with respect to the distance to the analyzer makes possible direct sampling of the plasma. The position of the entrance slits of the analyzer restricts the charge-exchange neutrals to those generated in a limited region of the plasma, a column about 1 centimeter high and 1 centimeter wide across the plasma diameter. Only ions moving perpendicular to the toroidal magnetic field are sampled. The energetic charge-exchanged neutral atoms are then re-ionized in a nitrogen gas cell at 13 pascals (0. 1 torr) of pressure and analyzed by a set of $90^{\circ}$ electrostatic deflector plates. The geometry of these plates requires that a re-ionized particle have an energy of 10 times the voltage across the plates per unit charge in order to be detected by the scintillator and photomultiplier tube at the output of the electrostatic deflector plates. The photomultiplier detector counts individual particles, and the signal is integrated and plotted as a function of energy in an X-Y recorder. From these raw data, several corrections are made as described in reference 16 to convert the raw data to ion energy distribution functions. These distribution functions are then compared with a Maxwellian distribution by a least-squares procedure to determine a best-fitting ion temperature. The raw data be- 
low 2000 electron volts are not used in the fit because the sensitivity of the scintillator crystal is not known with certainty below this energy for either helium or deuterium gas.

Two ion energy spectra taken with the charge-exchange neutral particle analyzer in helium gas are shown in figure 18. Figure 18(a) shows the measured data and the best-fitting Maxwellian distribution with an ion kinetic temperature of 2510 electron volts. Figure 18(b) shows an example in which the kinetic temperature was 1820 elec-

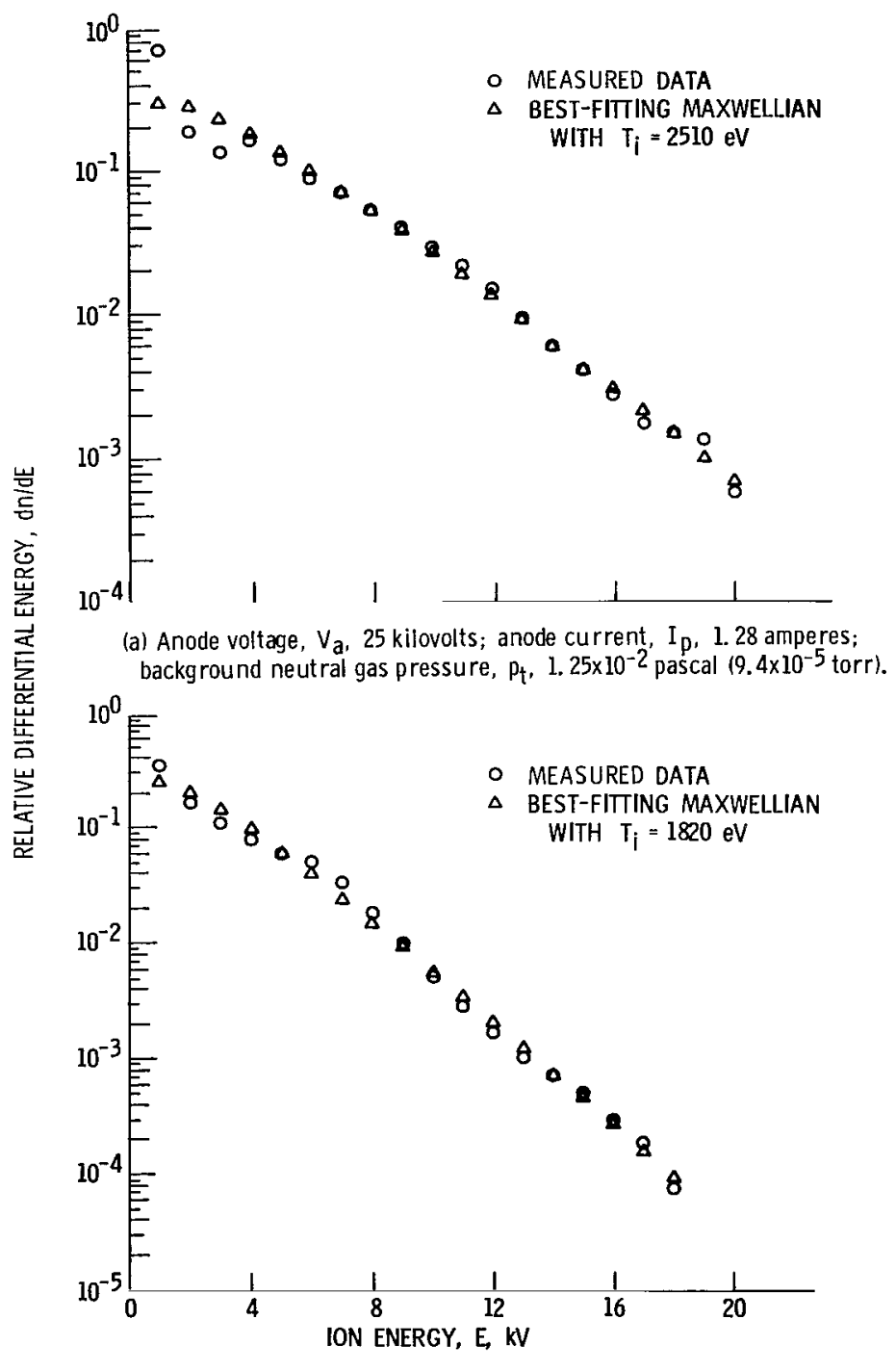

(b) Anode vnltage, $v_{a}, 15$ kilovolts; anode current, $I_{p}, 0.945$ ampere; background neutral gas pressure, $p_{\mathfrak{t}}, 1.33 \times 10^{-2}$ pascal $\left(10^{-4}\right.$ torr).

Figure 18. - Perpendicular ion energy distribution functions measured with charge-exchange neutral particle analyzer in helium gas. Maximum magnetic field strength, $B_{\max }, 24$ teslas. 
tron volts and the ion energy distribution could be followed even further along the Maxwellian tail. In figure 18(b) the Maxwellian tail can be followed out to 18 kilovolts, although the anode voltage was only 15 kilovolts. This demonstrates that the ion population is not directly heated by acceleration to the applied voltage in sheaths but that thermalization takes place. This observation is typical of all those cases at high magnetic fields for which the ion energy distribution function can be followed beyond the anode voltage. Data were also taken in deuterium gas and were reduced on the assumption that only $\mathrm{D}^{+}$ions are detected by the neutral particle analyzer. These measurements yielded ion kinetic temperatures from 160 to 1785 electron volts. The nature of the ion energy distribution function was investigated as a function of discharge operating conditions. An interesting trend became apparent, particularly when magnetic field was the independent variable. It was found that the ion energy distribution functions could be classified into four mutually exclusive descriptive categories, examples of which are illustrated in figure 19. In this figure a characteristic example of the ion energy distribution function is shown for each category along with the best-fitting Maxwellian distribution where this is appropriate. The most common distribution function best fits a single-slope Maxwellian distribution and is most prevalent at high magnetic fields, above $\mathrm{B}_{\max } \approx 2.0$ teslas. An example of the single-slope Maxwellian distribution is shown in figure 19(a). The reduced data closely fit a Maxwellian distribution with kinetic temperature of 697 electron volts over four orders of magnitude in the relative number density, or more than nine energy e-folding lengths, in spite of the fact that the analyzer sampled ions across the entire diameter of the plasma.

The next most frequent type of distribution function was the two-slope distribution illustrated in figure 19(b). These two-slope distribution functions are most prevalent at intermediate magnetic fields $\left(0.5 \leq \mathrm{B}_{\max } \leq 2.0\right.$ teslas). Such a two-slope distribution could result from the analyzer sampling the center and the sheath of the plasma, with each region of the plasma having a different ion temperature. Or the higher temperature component could represent $\mathrm{D}_{2}{ }^{+}$, which is hotter because of its higher mass, since all ions have a constant $(\vec{E} \times \vec{B}) / B^{2}$ drift velocity.

The third type of ion energy distribution function is the two-humped distribution illustrated in figure 19(c). Englert, Reinmann, and Lauver (ref. 24) have shown that distribution functions of this nature can arise when the plasma consists of two species (such as $\mathrm{D}^{+}$and $\mathrm{D}_{2}{ }^{+}$) at a common kinetic temperature with both species drifting in azimuth with a velocity comparable to the ion thermal velocity. In figure 19(c), neither hump fits a drift-free Maxwellian distribution, and it is clear that under these conditions the plasma as a whole is far from equilibrium. This type of distribution function tended to occur at pressures above $4.7 \times 10^{-3}$ pascal $\left(3.5 \times 10^{-5}\right.$ torr $)$ and at midplane magnetic fields less than 0.2 tesla $\left(B_{\max }<0.5\right.$ tesla). The second hump occurs at an energy approximately twice that of the first maximum. According to reference 24 the 


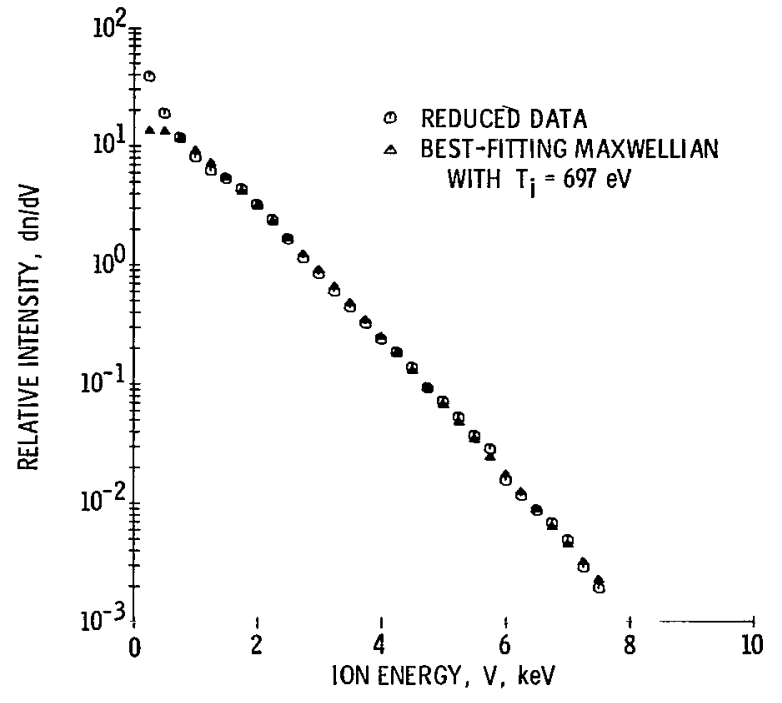

(a) Maxwellian distribution; anode voltage, $V_{a}, 8$ kilovolts.

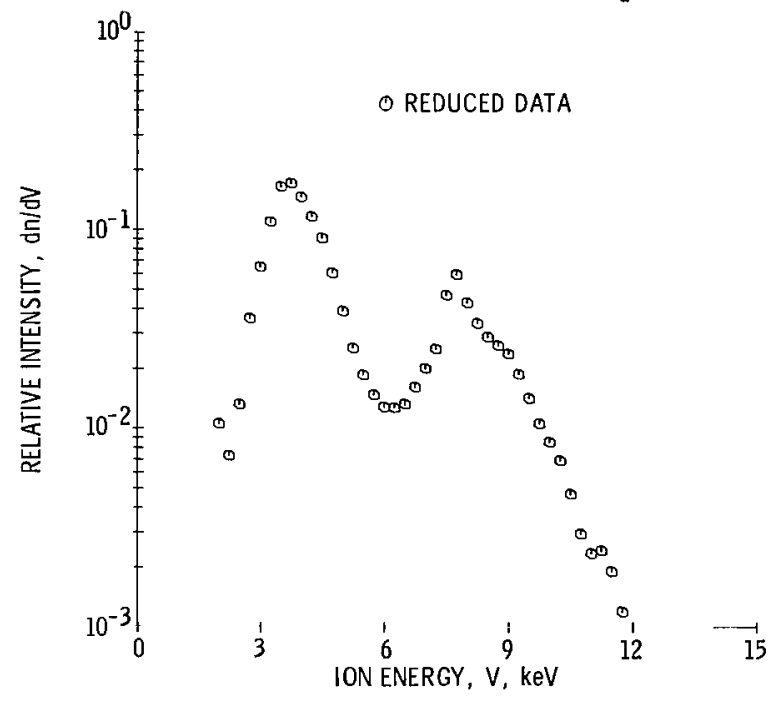

(c) Two-humped distribution; anode voltage, $V_{a}$, 7.5 kilovolts.

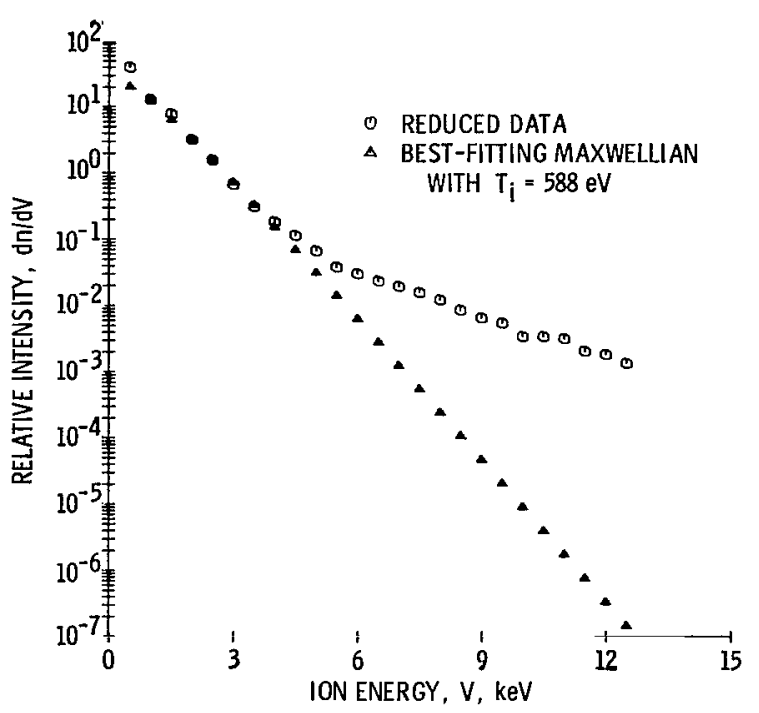

(b) Two-slope distributions; anode voltage, $V_{a}, 12.5$ kilovolts.

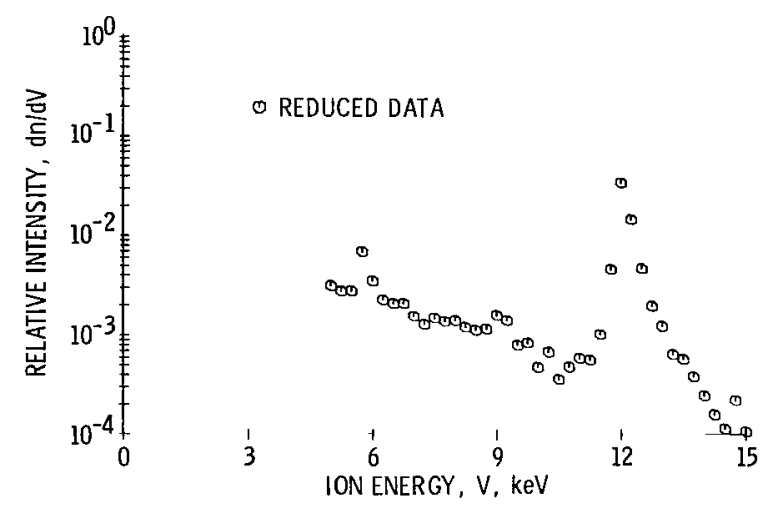

(d) Peak-dominated distribution; anode voltage, $V_{a}$, 12.5 kilovolts.

Figure 19. - Four mutually exclusive types of ion energy distribution function observed in bumpy-torus plasma operating at low power in deuterium gas.

plasma at these operating conditions could consist of a Maxwellian plasma with an ion temperature of about 100 electron volts rotating at a high $(\overrightarrow{\mathrm{E}} \times \overrightarrow{\mathrm{B}}) / \mathrm{B}^{2} \mathrm{drift}$ velocity. The second hump may be due to $\mathrm{D}_{2}^{+}$rotating with the same drift velocity.

The final ion energy distribution is shown in figure 19(d). It is dominated by a single very large peak at the anode voltage of the plasma. These peaks tend to occur at pressures below $4.7 \times 10^{-3}$ pascal $\left(3.5 \times 10^{-5}\right.$ torr) and at midplane magnetic fields less than 0.2 tesla. In some cases, peaks occur at one-half and three-fourths of the anode 
voltage. The peak at one-half of the anode voltage could be due either to dissociation of $\mathrm{D}_{2}{ }^{+}$in the plasma or of $\mathrm{D}_{2}{ }^{+}$or neutral $\mathrm{D}_{2}$ in the gas cell.

As the magnetic field increases, the percentage of cases in which the ion energy distribution function best fits a Maxwellian distribution increases. This is fortunate because it is desirable in fusion applications that the ions be as Maxwellian as possible in order to reduce the number of reservoirs of free energy available to drive plasma instabilities.

\section{RADIAL PROFILES OF FLOATING POTENTIAL}

The electric fields acting on the plasma play an essential role in ion heating and may affect the plasma confinement and stability. Hence, it was considered desirable to obtain information about the radial profile of floating potential. Ion beam probing is the method of choice of such measurements, but only the conventional floating Langmuir probe technique was possible during these investigations. There are serious questions about the validity of floating potential measurements under the existing conditions of high ion temperature, strong magnetic field, and measurable disturbance of the plasma. It was nonetheless hoped that such measurements could at least provide qualitative information about whether the plasma as a whole was floating at a high potential and whether the electric field was pointing inward or outward in the vicinity of the plasma boundary.

Radial profiles of floating potential were taken in the bumpy-torus plasma with the hydraulically actuated Langmuir probe shown in figure 20. The length of travel of the probe tip could be adjusted continuously over a radial distance of 11.4 centimeters, with a dwell time of 0.7 second at each position along this travel length at which it was set.

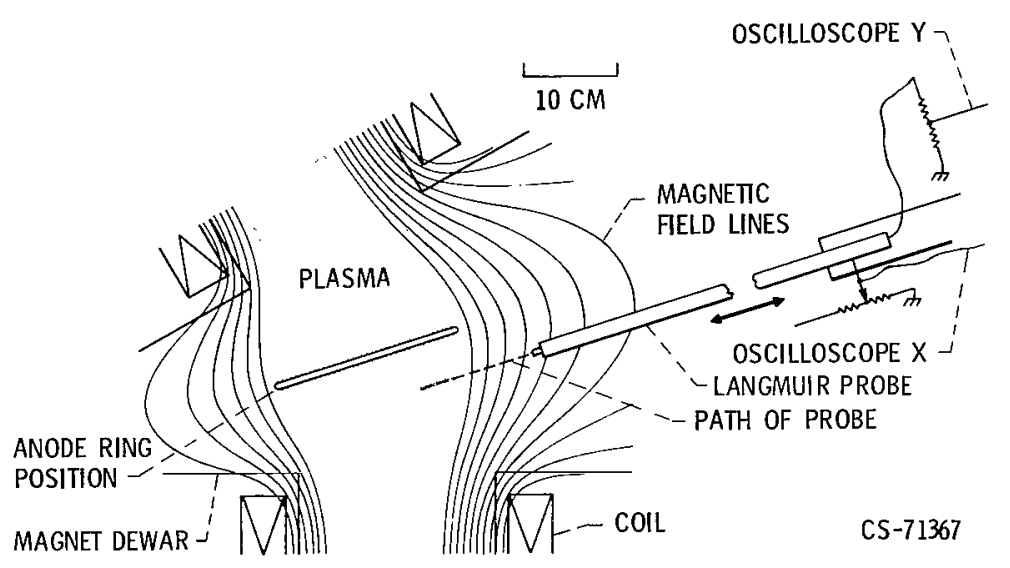

Figure 20. - Region of plasma sampled by Langmuir probe. 
The probe potential is allowed to float with a $100-$ megohm impedance to ground, which compares with an effective plasma resistance (obtained by dividing the anode voltage by the anode current) less than about 100 kilohms for these measurements.

The results of these measurements are shown in figure 21 for a plasma with positive potentials applied to all 12 anode rings. All data shown in figure 21 are in the lowpressure mode of operation except for the lowest curve at an anode voltage of 2.3 kilovolts, which is in the high-pressure mode. The plane of the probe motion lies 4.3 centimeters away from the plane of the adjacent anode ring. The probe was started from a position 7.5 centimeters outside the anode ring radius, and it penetrated approximately 3 centimeters into the plasma. The probe was started this far out to prevent its overheating and perturbing the plasma too greatly. No effect of the probe on the anode current was observed during the measurements shown in figure 21 . The results in figure 21 indicate that the plasma floats at a potential of at least 75 percent of the anode voltage and that the electric field points radially outward over its surface.

Results with a single anode $r$ ing are shown in figure 22 for the high-pressure mode

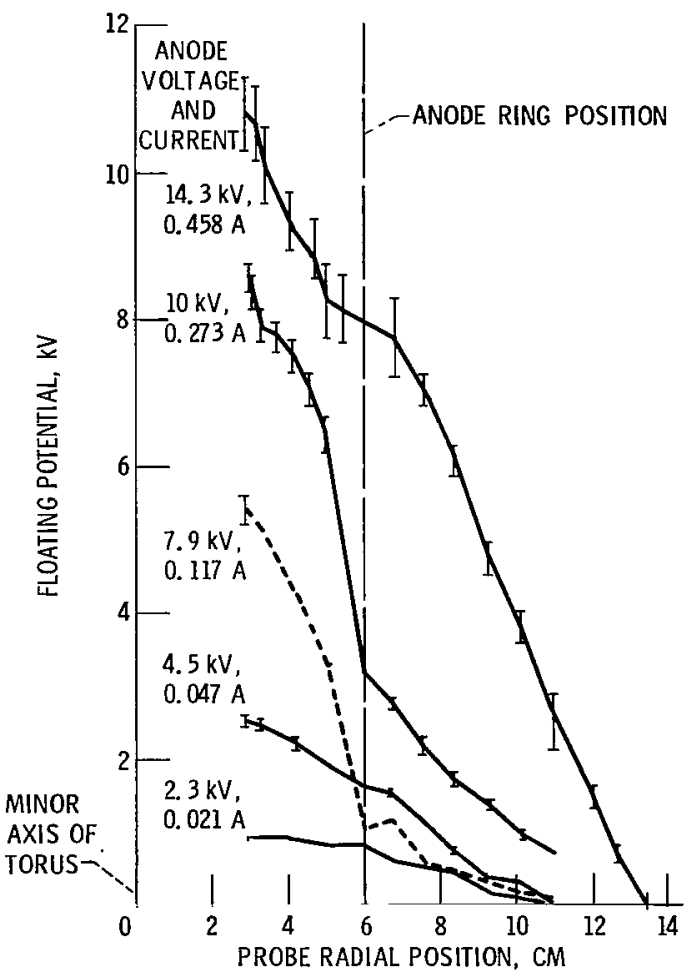

Figure 21. - Floating potential as function of probe position along radius 4.3 centimeters from magnetic midplane of sector 8 . Maximum magnetic field strength, $B_{\max }, 2.4$ teslas; deuterium background pressure, $p_{t}, 3.6 \times 10^{-3}$ pascal $\left(2.7 \times 10^{-5}\right.$ torr). 


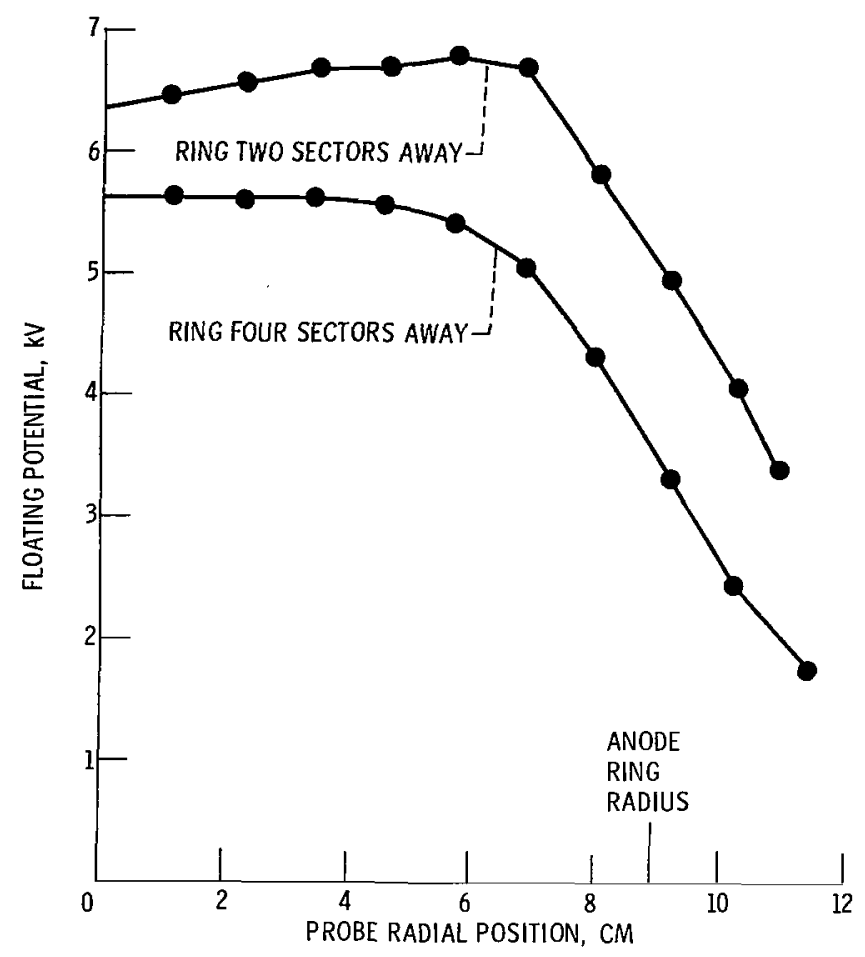

Figure 22. - Radial floating potential profile as a result of changing position of single anode ring from two sectors away to four sectors away from sector containing probe - high-pressure mode. Anode voltage, $V_{a}, 7$ kilovolts; background neutral gas (helium) pressure, $p_{t}, 1.1 \times 10^{-2}$ pascals $\left(8.3 \times 10^{-5}\right.$ torr); maximum magnetic field strength, $B_{\max }, 2.4$ teslas.

and in figure 23 for the low-pressure mode. The anode ring was first placed two sectors away from the sector in which the probe was located; then it was removed and placed four sectors away. As the anode ring was moved away from the probe, the potential in the probe sector decreased in both the high- and low-pressure modes. The decrease was much more dramatic in the low-pressure mode. The high-pressure-mode results (fig. 22) indicate that the plasma ring floats at a positive potential well above ground. The low-pressure-mode results, with only one anode ring (fig. 23), should be interpreted cautiously since, unlike the high-pressure-mode condition, the anode current changed by as much as 50 percent when the probe was inserted into the plasma. It is also the case that misalinement of the single anode ring used in figures 22 and 23 could have affected the results. It appears justified to conclude from these measurements that when positive potentials are applied to the midplane electrode rings, the plasma floats at a high positive potential and reaches at least 50 to 80 percent of the anode voltage on the plasma axis. It also appears justified to conclude that, except in the vicinity of the anode sheath, the electric field created by this floating potential points radially outward from the plasma. 


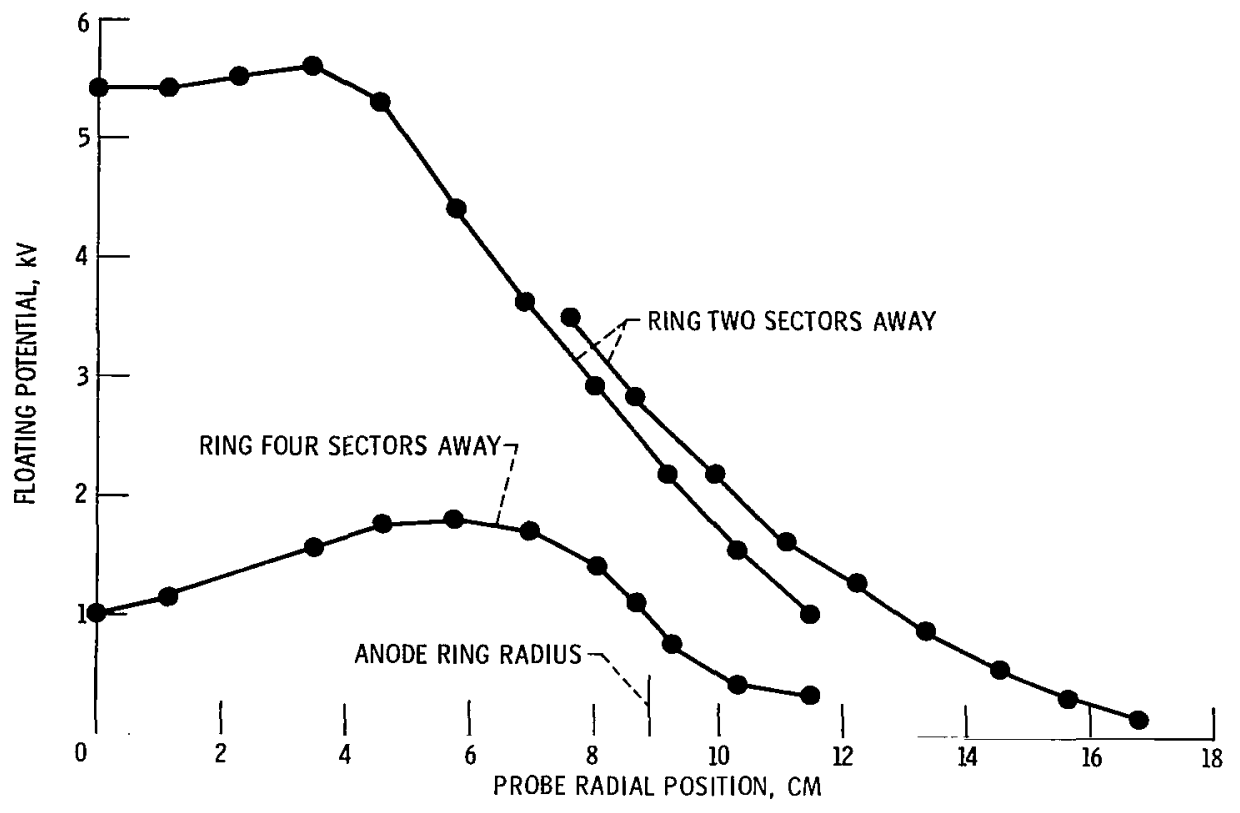

Figure 23. - Radial floating potential profile as a result of changing position of single anode ring from two sectors away to four sectors away from sector containing probe - low-pressure mode. Anode voltage, $v_{a}, 20$ kilovolts; background neutral gas (helium) pressure, $p_{t}, 3.7 \times 10^{-3}$ pascals $\left(2.8 \times 10^{-5}\right.$ torr); maximum magnetic field strength, $B \max , 2.4$ teslas. (The two curves representing the twosectors-away case show effect on potential profile of moving grounded probe support shaft $7.6 \mathrm{~cm}$ radially outward from plasma. )

\section{SPECTROSCOPICALLY DETERMINED RADIAL PROFILES OF ELECTRON}

\section{DENSITY AND TEMPERATURE}

Spatially averaged and time-averaged electron temperature and relative number density have been measured spectroscopically over a range of discharge operating conditions in the NASA Lewis bumpy-torus plasma. Observations in pure helium gas have been reported elsewhere by Richardson (ref. 19). The electron temperature is measured from the ratio of selected spectral line intensities emitted by a small admixture of helium gas. Relative number densities are deduced from the helium spectral line intensities and measured electron temperatures.

The spectroscopic measurements were made with a 1.5-meter Fastie-Ebert spectrometer. A thin horizontal chord through the plasma perpendicular to the magnetic field was viewed through a beam rotator and imaging optics, as shown in figure 24 . The measurements were made at the magnetic mirror midplane in the region between a set of double anode rings spaced 3 centimeters apart. Chords at different vertical positions 


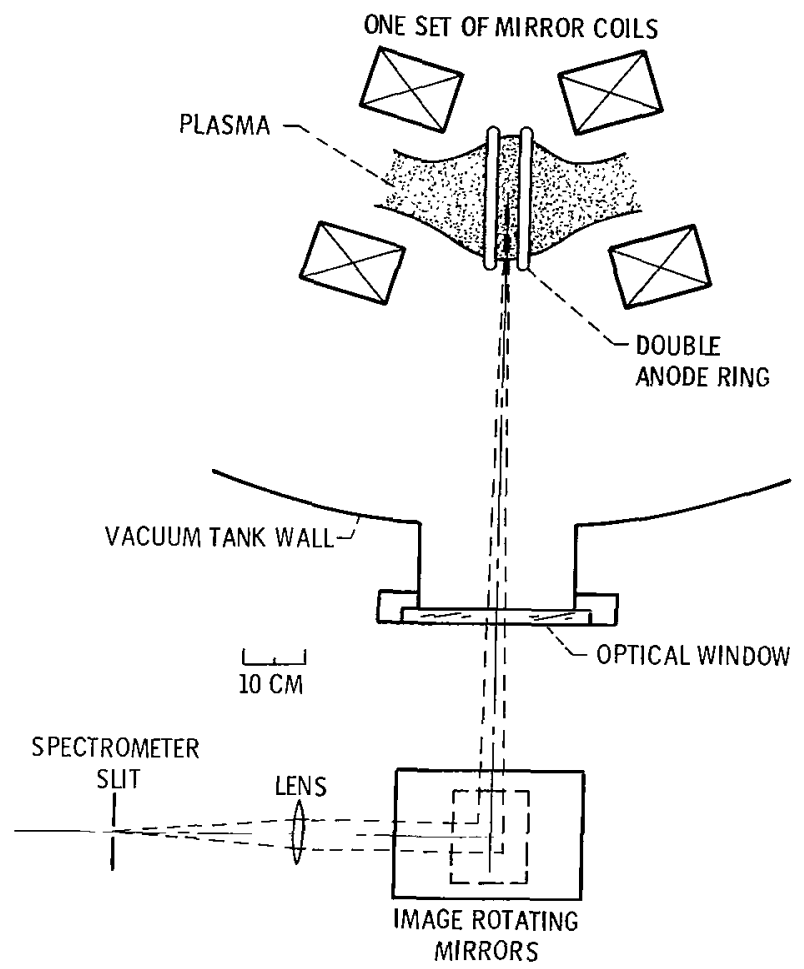

Figure 24. - Schematic drawing of spectroscopic apparatus used to take electron temperature and relative number density and to make radial profiles of these quantities.

were observed by translation of the lens perpendicular to the optical axis. A standard lamp was used to determine the spectral response of the entire system.

In order to produce observable helium spectral lines, a small amount of helium was added to the background deuterium gas. A constant helium flow rate equivalent to a background pressure of $9.3 \times 10^{-4}$ pascal $\left(7 \times 10^{-6}\right.$ torr $)$ based on an ionization gage factor of 5.55 was used for most runs. It was assumed that the pumping speed of the system was constant for helium gas. The deuterium background pressure was varied between 1. $3 \times 10^{-3}$ and $1.1 \times 10^{-2}$ pascal $\left(10 \times 10^{-6}\right.$ and $8 \times 10^{-5}$ torr $)$ based on a gage factor of 2.65 . A few runs were taken at deuterium pressures of $5.3 \times 10^{-4}$ to $1.3 \times 10^{-3}$ pascal $\left(4 \times 10^{-6}\right.$ to $10 \times 10^{-6}$ torr) for which a helium background pressure of $6.7 \times 10^{-4}$ pascal $\left(5 \times 10^{-6}\right.$ torr) was used. The percentage of helium mixture was varied over the range used experimentally, and the electron temperature was not sensitive to the fraction of helium mixed with the deuterium until this fraction became greater than about 50 percent.

Electron temperatures were measured by the helium line ratio technique (refs. 25 and 26). This technique uses the marked difference in electron energy dependence of the excitation cross section between the singlet and triplet series of helium and between the helium $I$ and neutral helium lines. The intensity of a spectral line $I_{j k}$ due to a 
transition $j \rightarrow k$ in the case of single-step electron excitation and spontaneous decay is

$$
I_{j k}=n_{0} n_{e}\left\langle f_{j k}(v) v\right\rangle \sigma_{j k}
$$

for the corona model (refs. 25 and 26), where $n_{0}$ and $n_{e}$ are the neutral helium and electron number densities, respectively. The variable $f_{j k}(v)$ is the apparent excitation cross-sectional shape function normalized to its maximum and is a function of the electron velocity $v$. The angular brackets indicate an average over the assumed electron energy distribution function that is taken to be Maxwellian, and $\sigma_{j k}$ is the maximum apparent excitation cross section. The ratio of the intensity of two spectral lines $\mathrm{I}_{\mathrm{jk}} / \mathrm{I}_{\mathrm{lm}}$ is independent of electron density and is a function of electron temperature if the shape functions $f_{j k}(v)$ and $f_{l m}(v)$ are dissimilar. Sovie (ref. 25) has suggested that transitions from the ${ }^{1} \mathrm{~S}$ and ${ }^{3} \mathrm{~S}$ states be used since the cross section for excitation to these states has been shown to be insensitive to background neutral gas pressure and, hence, to multistep processes involving neutrals, which would invalidate equation (2).

The excitation coefficients $\sigma_{j k}\left\langle f_{j k}(v) v\right\rangle$ and appropriate ratios as functions of electron temperature were numerically computed up to an electron temperature of 200 electron volts for the $2^{3} \mathrm{P}-5^{3} \mathrm{~S}(412.1 \mathrm{~nm}), 2^{3} \mathrm{P}-4^{3} \mathrm{~S}(471.3 \mathrm{~nm}), 2^{1} \mathrm{P}-4{ }^{1} \mathrm{~S}(504.8 \mathrm{~nm})$, and $2^{1} \mathrm{P}-5^{1} \mathrm{~S}(443.8 \mathrm{~nm})$ transitions of neutral helium by using the shape functions of references 27 and 28 and I. D. Lattimer (private communication). The $\mathrm{He}^{+}(468.6 \mathrm{~nm}$ ) transition excitation coefficient was calculated by using the shape functions of references 25 to 30 . For all lines the maximum cross sections $\sigma_{j k}$ of reference 26 were used.

The relative electron density $\hat{\mathrm{n}}_{\mathrm{e}}$ can be determined from the measured electron temperature and the relation

$$
\hat{\mathrm{n}}_{\mathrm{e}}=\frac{\hat{\mathrm{n}}_{\mathrm{e} 0} \mathbf{I}_{\mathrm{jk}}}{\mathrm{n}_{0}\left\langle\mathrm{f}_{\mathrm{jk}}(\mathrm{v}) \mathrm{v}\right\rangle \sigma_{j k}}
$$

where $I_{j k}$ is the observed relative spectral line intensity and $\hat{n}_{e 0}$ is an arbitrary normalizing constant that was the same for all data presented in this report. This relation is useful for determining radial relative density profiles and observing the change in density with operating conditions of the discharge.

Relative helium line intensities spatially averaged across a chord through the plasma 2 centimeters from the edge of the plasma have been measured while the discharge operating conditions were systematically varied. The $2^{3} \mathrm{P}-5^{3} \mathrm{~S}(412.1 \mathrm{~nm})$ and $2^{3} \mathrm{P}-4^{3} \mathrm{~S}(471.3 \mathrm{~nm})$ triplet series transitions of neutral helium were observed, as well as the 468.6-nanometer line of helium $I I$ under the higher electron temperature conditions. With the discharge operating at a fixed voltage and constant deuterium pressure, 
the spectrometer was manually peaked on each spectral line, and the light intensity was measured with the helium supply on and off - the difference being the spectral line intensity. No change in the background light level was detectable between the helium-on and helium-off conditions, and no pronounced deuterium or impurity lines interfered with the helium lines. Typical spectroscopic data at a set discharge operating condition are presented in figure 25. Wavelength scans of the spectrum near the line with and without helium are shown, as is the peak intensity as a function of time for about 1 minute of operation. The noise level for the chosen conditions is about average.

Figure 26 shows sample vertical scans of the 504. 8-nanometer helium line intensity. The horizontal scale is the vertical distance of the chord from the center of the discharge, and the vertical scale is the relative line intensity. A vertical scan is shown for the low - and high-pressure operating modes of the discharge. Forty-point Abel inversions (ref. 31) have been made of the vertical profiles of the spectral lines for various operating conditions of the discharge. The effect of azimuthal drift and nonaxisymmetry due to toroidal curvature has been neglected.

Radial profiles of the electron temperature and relative number density were taken for various conditions in the high- and low-pressur e modes of operation. Characteristic sets of four of these profiles are shown in figures 27 and 28, and the corresponding operating conditions are specified in table III. These conditions are also indicated by the points $A$ to $D$ on the current-voltage plot for deuterium gas (fig. 8(a)). The mode transitions and current-voltage curves for the data of table III are slightly displaced from those of figure $8(\mathrm{a})$ as a result of the admixture of helium gas for spectroscopic purposes. Case $A$ is in the high-pressure mode, somewhat away from the mode transition boundary; cases $B$ and $C$ are at essentially the same background pressure but on adjacent sides of a mode transition; and case $D$ is in the low-pressure mode away from the mode transition. The scatter of adjacent points about the trend line is due to numerical errors introduced by the Abel inversion procedure. These profiles exhibit three features that are characteristic of a large majority of the profile data taken:

(1) The electron temperature profiles are relatively flat across the plasma diameter.

(2) The electron temperatures are higher at the outer boundary of the plasma, in the vicinity of the anode sheath.

(3) The temperatures are higher in the low-pressure mode than in the high-pressure mode.

Radial profiles of relative electron number density are shown in figure 28 for the four operating conditions of table III. The scatter of adjacent points about the trend line is again due to numerical errors introduced by the Abel inversion procedure. These density profiles exhibit several features that are characteristic of the profile data taken: (1) In the low-pressure mode, the density profile is relatively flat in the plasma in- 


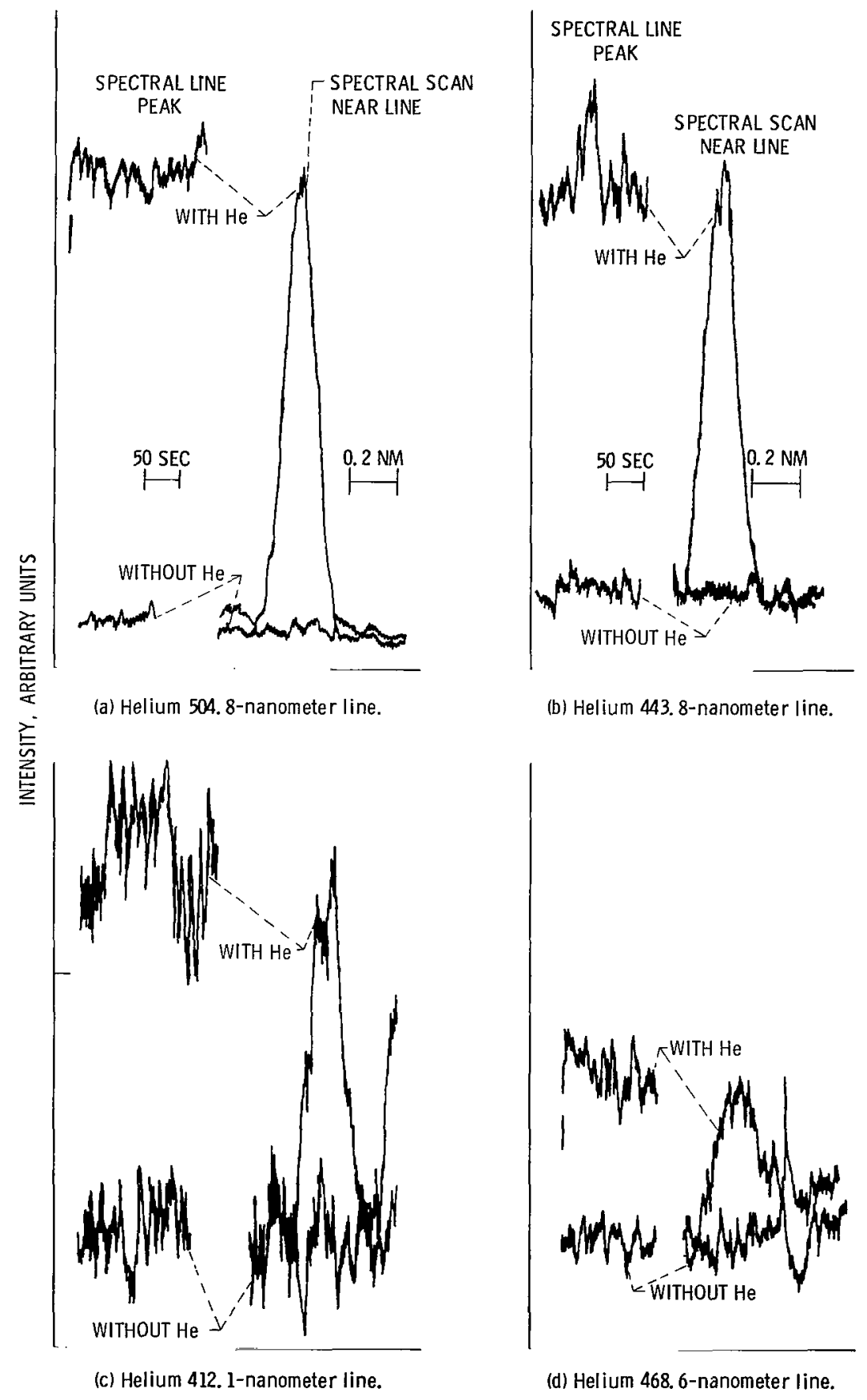

Figure 25. - Typical spectral peak, background noise, and method of measuring peak height. (Spectral peak was scanned in wavelength, and then peak intensity was measured as function of time.) 


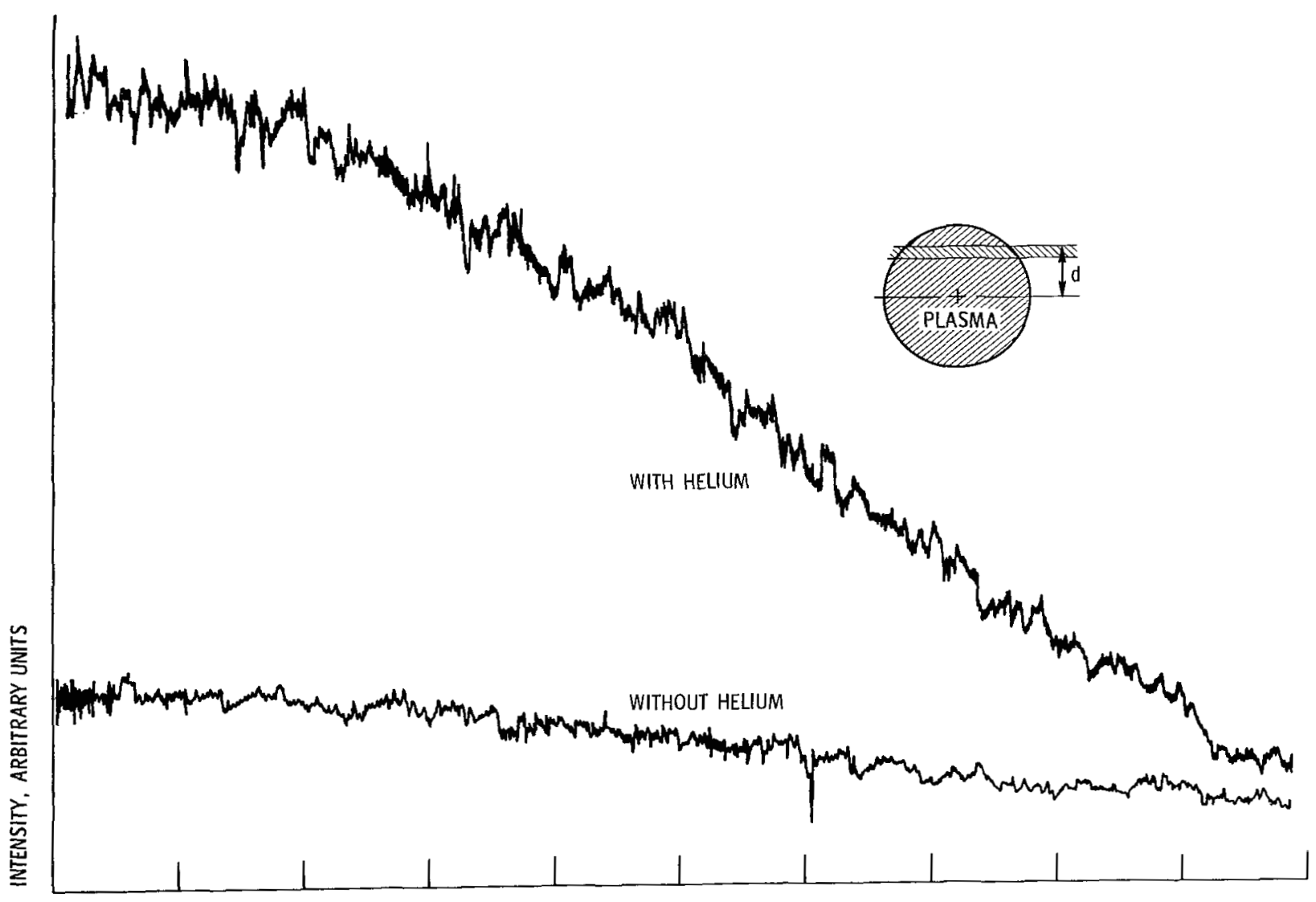

(a) High-pressure mode. Anode voltage, $V_{a}, 5$ kilovolts; anode current, $I_{p}, 260$ mill iamperes; background neutral gas pressure, $p_{f^{\prime}}$ $4.4 \times 10^{-3}$ pascal $\left(3.3 \times 10^{-5}\right.$ torr).

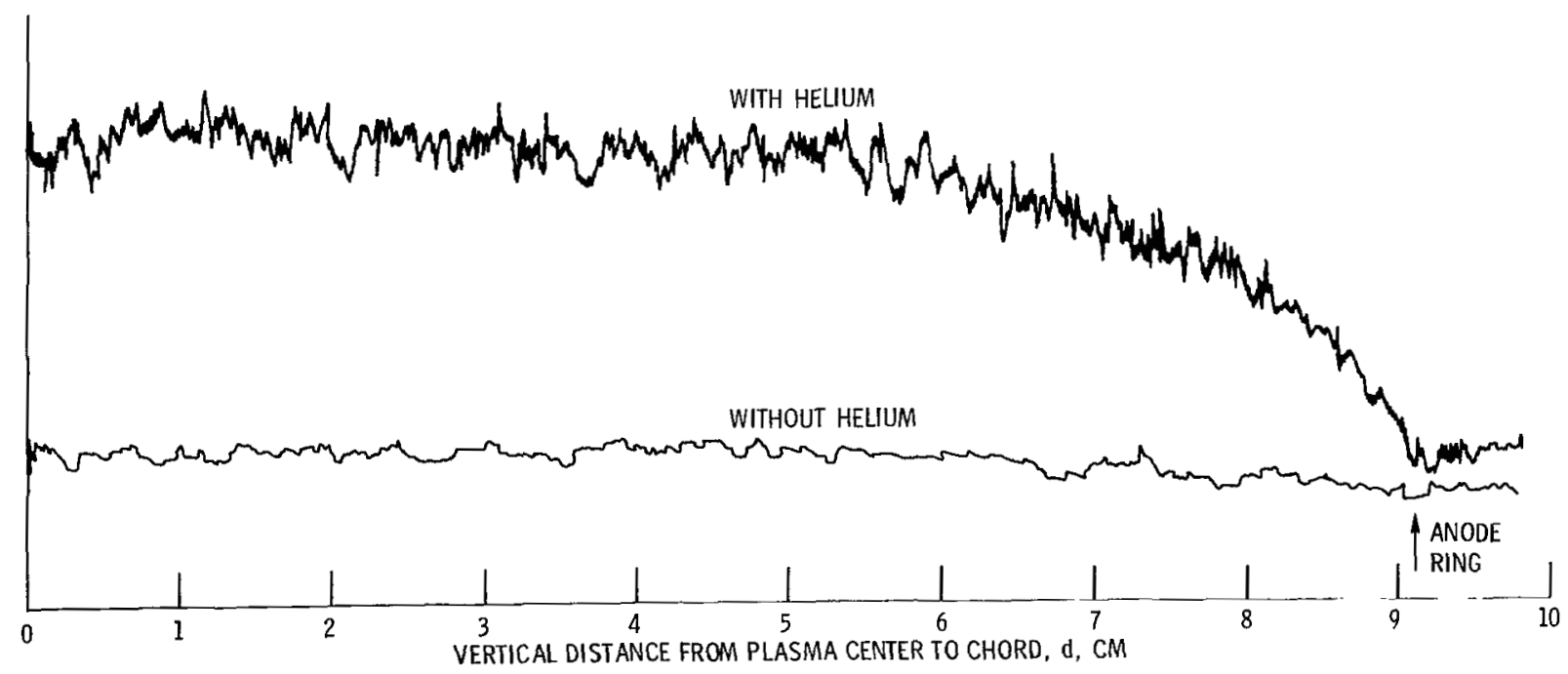

(b) Low-p ressure mode. Anode voltage, $V_{a}, 9$ kilovolts; anode current, $I_{p}, 200$ milliamperes; background neutral gas pressure, $p_{t}$, 3.7 $\times 10^{-3}$ pascal $\left(2.8 \times 10^{-5}\right.$ torr).

Figure 26. - Raw data showing radial scan of amplitude of 504. 8-nanometer helium line for the two operating modes of the discharge. Maximum magnetic field strength, $B_{\text {max }}, 2.4$ teslas.

34 


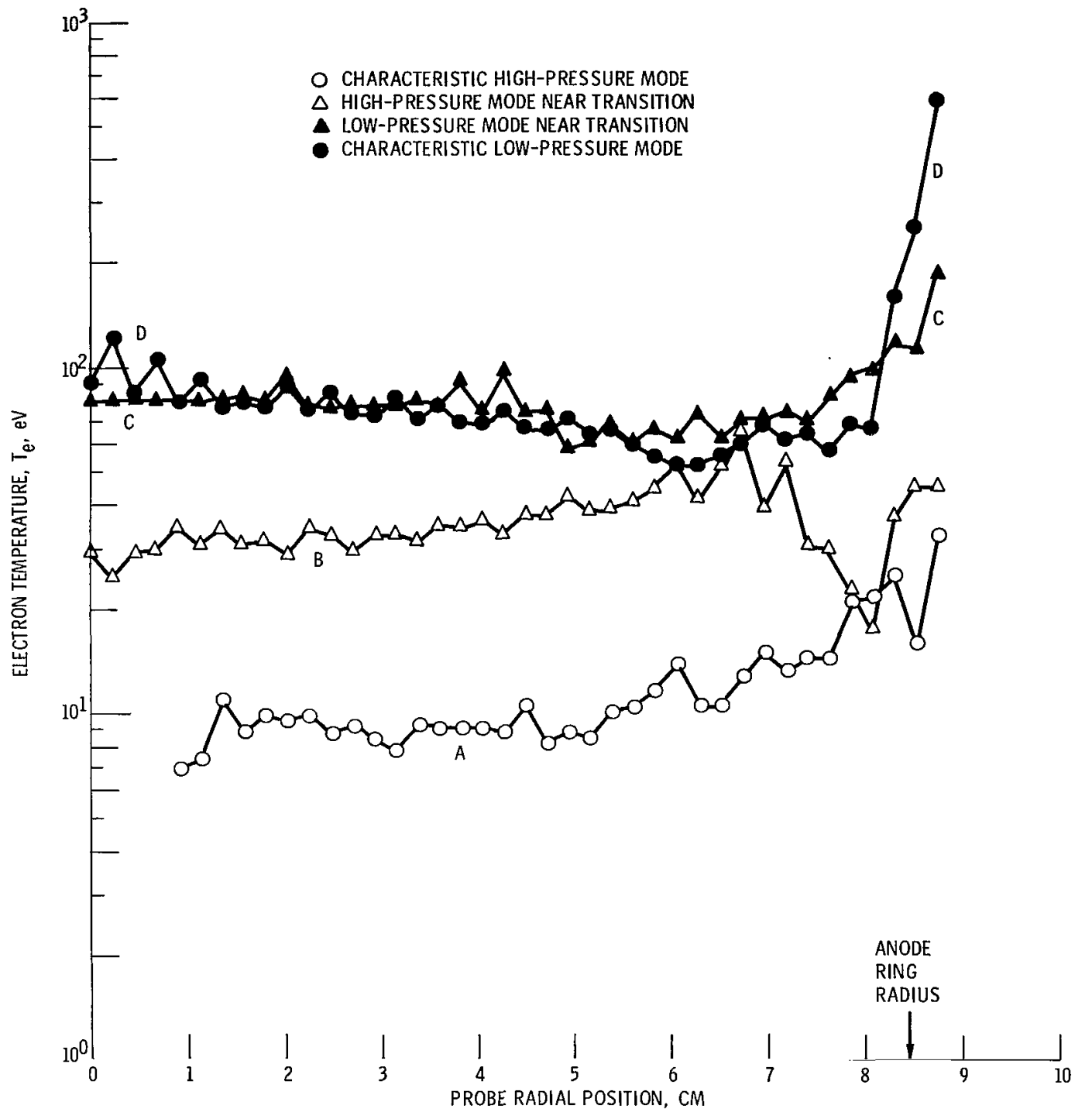

Figure 27. - Electron temperature as function of probe radial position in deuterium gas for four operating conditions designated $A$ to $D$ in figure $8(a)$ and table III.

terior but is enhanced in an annular ring associated with the anode sheath.

(2) In the high-pressure mode, the density peaks on the axis and falls off by about a factor of 10 at the anode sheath.

(3) The average densities are higher in the high-pressure mode than in the lowpressure mode, if other conditions are approximately constant, as they are in cases B and $\mathrm{C}$.

(4) If other factors are the same, the densities tend to increase with increasing anode current, anode voltage, and neutral gas pressure.

(5) Ionization occurs throughout the plasma volume in the high-pressure mode but is 


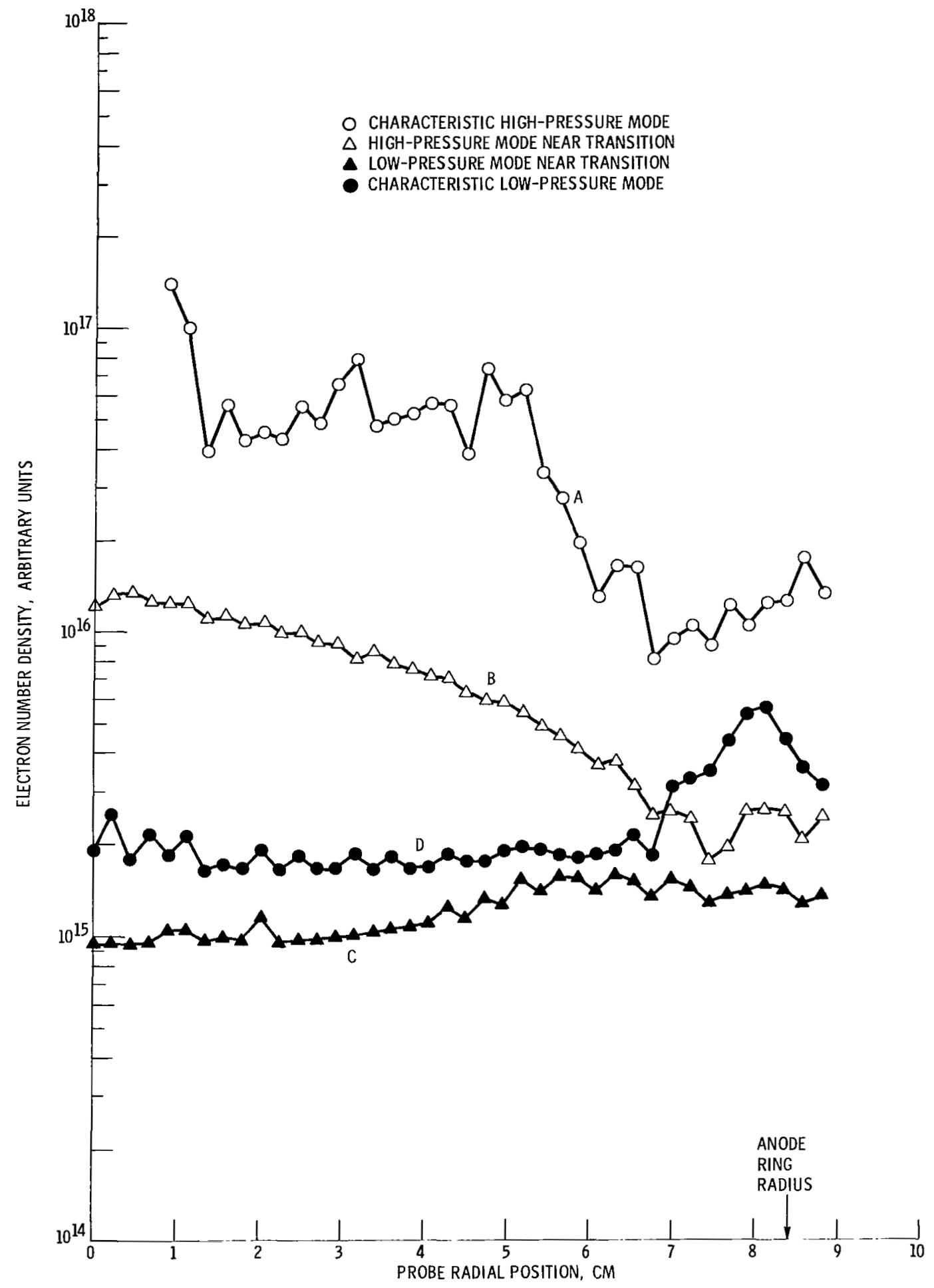

Figure 28. - Relative electron number density as function of probe radial position in deuterium gas for four operating conditions designated $A$ to $D$ in figure $8(a)$ and table III. 
TABLE III. - OPERATING CONDITIONS FOR RADIAL ELECTRON DENSITY AND TEMPERATURE PROFILES

\begin{tabular}{|c|c|c|c|c|}
\hline \multirow[t]{4}{*}{ Operating condition } & \multicolumn{4}{|c|}{ Location in figure $8(a)$} \\
\hline & A & $\mathrm{B}$ & $\mathrm{C}$ & $\mathrm{D}$ \\
\hline & \multicolumn{4}{|c|}{ Mode } \\
\hline & High pressure & $\begin{array}{l}\text { High pressure (near } \\
\text { transition) }\end{array}$ & $\begin{array}{c}\text { Low pressure (near } \\
\text { transition) }\end{array}$ & Low pressure \\
\hline Tank pressure, $p_{t}, p_{a}$ (torr) & $1.0 \times 10^{-2}\left(7.6 \times 10^{-5}\right)$ & $3.5 \times 10^{-3}\left(2.6 \times 10^{-5}\right)$ & $3.2 \times 10^{-3}\left(2.4 \times 10^{-5}\right)$ & $2.3 \times 10^{-3}\left(1.7 \times 10^{-5}\right)$ \\
\hline Anode voltage, $\mathrm{V}_{\mathrm{a}}, \mathrm{kV}$ & 10 & 6 & 9 & 15 \\
\hline Anode current, $I_{p}, A$ & 1.36 & 0.35 & 0.20 & 0.27 \\
\hline Ion kinetic temperature, $\mathrm{T}_{\mathrm{i}}$, eV & 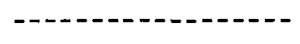 & 462 & 634 & 670 \\
\hline Maximum magnetic field strength, $\mathrm{B}_{\max }, \mathrm{T}$ & 2.4 & 2.4 & 2.4 & 2.4 \\
\hline
\end{tabular}


localized in the anode sheath in the low-pressure mode (ref. 19).

\section{PARTICLE TRANSPORT IN THE BUMPY-TORUS PLASMA}

The present experiment uses a modified Penning discharge in combination with a bumpy-torus magnetic field. It is to be expected that the confinement properties of this combination will differ significantly from those of other concepts such as Tokamaks or Stellarators. These concepts rely exclusively on magnetic fields to confine the plasma, but the Penning discharge - bumpy-torus combination is intended to use both electric and magnetic fields for plasma heating and confinement. Conventional containment concepts based on pure magnetic confinement have exhibited ion containment times that are proportional to the magnetic field (Bohm diffusion) or that vary as $\mathrm{B}^{2}$ (variations of classical diffusion). It is, therefore, of interest to see whether the Lewis bumpy torus exhibits similar behavior.

The steady-state current flowing to the Penning anode rings represents the net number of coulombs per second being lost from the entire plasma and can be written

$$
I_{p}=\frac{e n_{e} V_{p}}{\tau_{c}}
$$

where $V_{p}$ is the plasma volume, $n_{e}$ is the time-and space-averaged electron numbar density, and $\tau_{c}$ is the net charge residence time. Since the ions and electrons move across the magnetic field lines to complete the electrical circuit to the power supply, normally $I_{p}$ should be a function of magnetic field and should be governed by classical or Bohm diffusion. Studying whether the anode current is a function of magnetic field while discharge parameters other than the electron number density remain constant can provide a rough measure of the variation of the net charge residence time $\tau_{c}$ with magnetic field.

Figures 29(a), (b), and (c) show the current-voltage characteristics of the bumpytorus plasma for the same eight values of deuterium gas pressure and for maximum field strengths of $2.4,1.43$, and 0.48 tesla. A comparison of these characteristic curves shows that the sharp mode transition gradually disappears as the magnetic field is reduced, until the curves are continuous at 0.48 tesla. It is also apparent that the voltage-current curve for a given pressure is only weakly dependent on magnetic field. Spectroscopic measurements showed that the relative electron number density typically did not vary by more than a factor of 2 over this range of magnetic fields. If $n_{e}$ is regarded as approximately constant, Bohm diffusion would have predicted a shift of a factor of 5 in going from figure $29(\mathrm{a})$ to (c), and classical diffusion varying as $\mathrm{B}^{2}$ would 


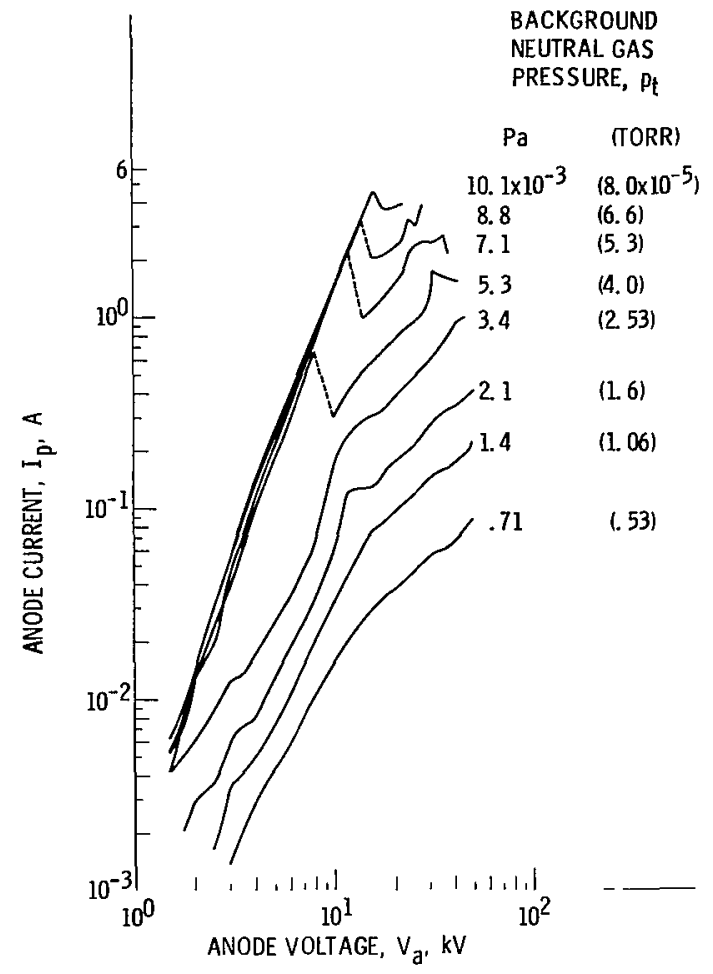

(a) Maximum magnetic field strength, $B_{\max }, 24$ teslas.
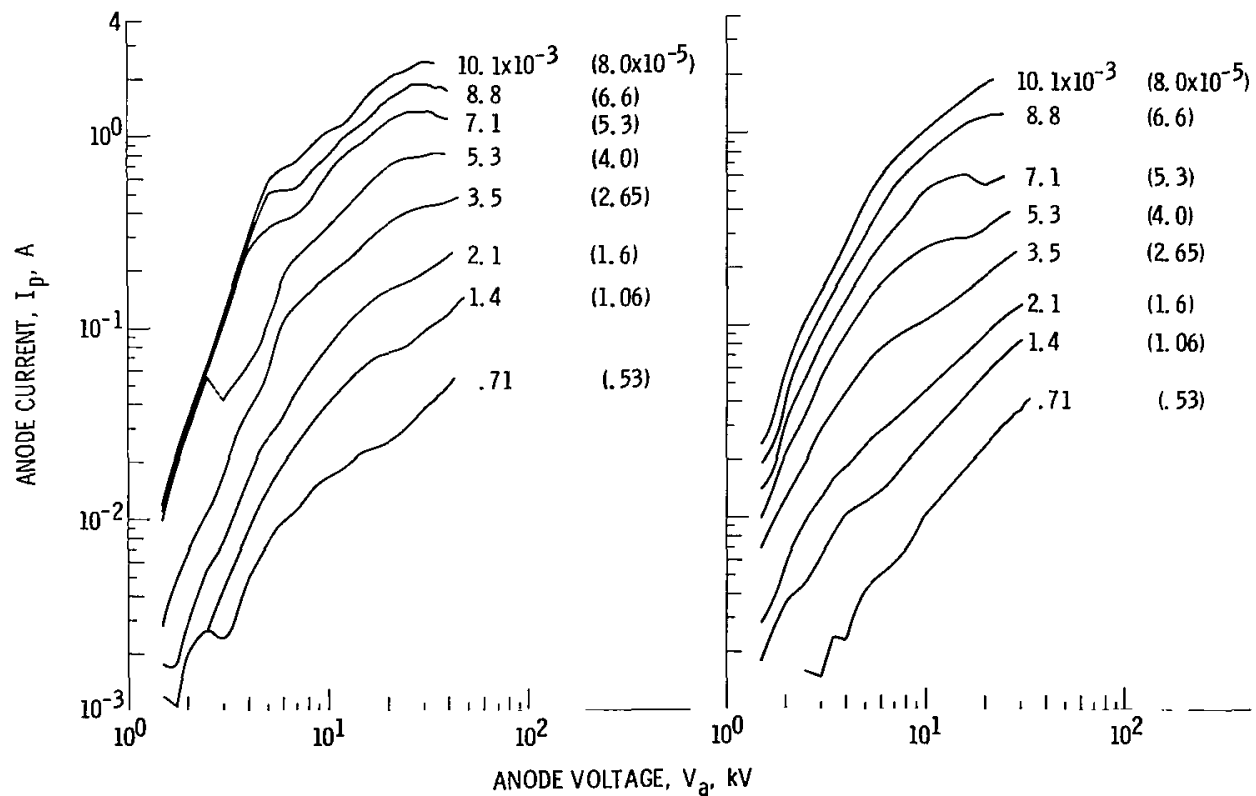

ANODE VOLTAGE, $V_{a}, k V$

(b) Maximum magnetic field strength, $B_{\text {max }}$ 1.43 teslas.

(c) Maximum magnetic field strength, $B_{\text {max }}$. 0.48 tesla.

Figure 29. - Current-voltage curves of bumpy-torus plasma in deuterium gas for various background neutral gas pressures. Number of anode rings, 12. 
have predicted a factor of $\mathbf{2 5}$ shift in any one of the characteristic curves.

This point can be illustrated more explicitly with the data shown in figure 30, in which the anode current is plotted as a function of magnetic field for three combinations of background pressure and anode voltage. Although the magnetic field varies over a factor of 10, the anode current varies only by about a factor of 2. A factor of 100 would have been anticipated on the basis of classical diffusion. Unfortunately, figures 29 and 30 are not conclusive because the relative electron number density was not measured simultaneously.

A second line of evidence that illustrates that particle transport is weakly dependent on magnetic field in the bumpy torus can be obtained from spectroscopic observations in the following manner: A time-and space-averaged continuity equation for the bumpy-

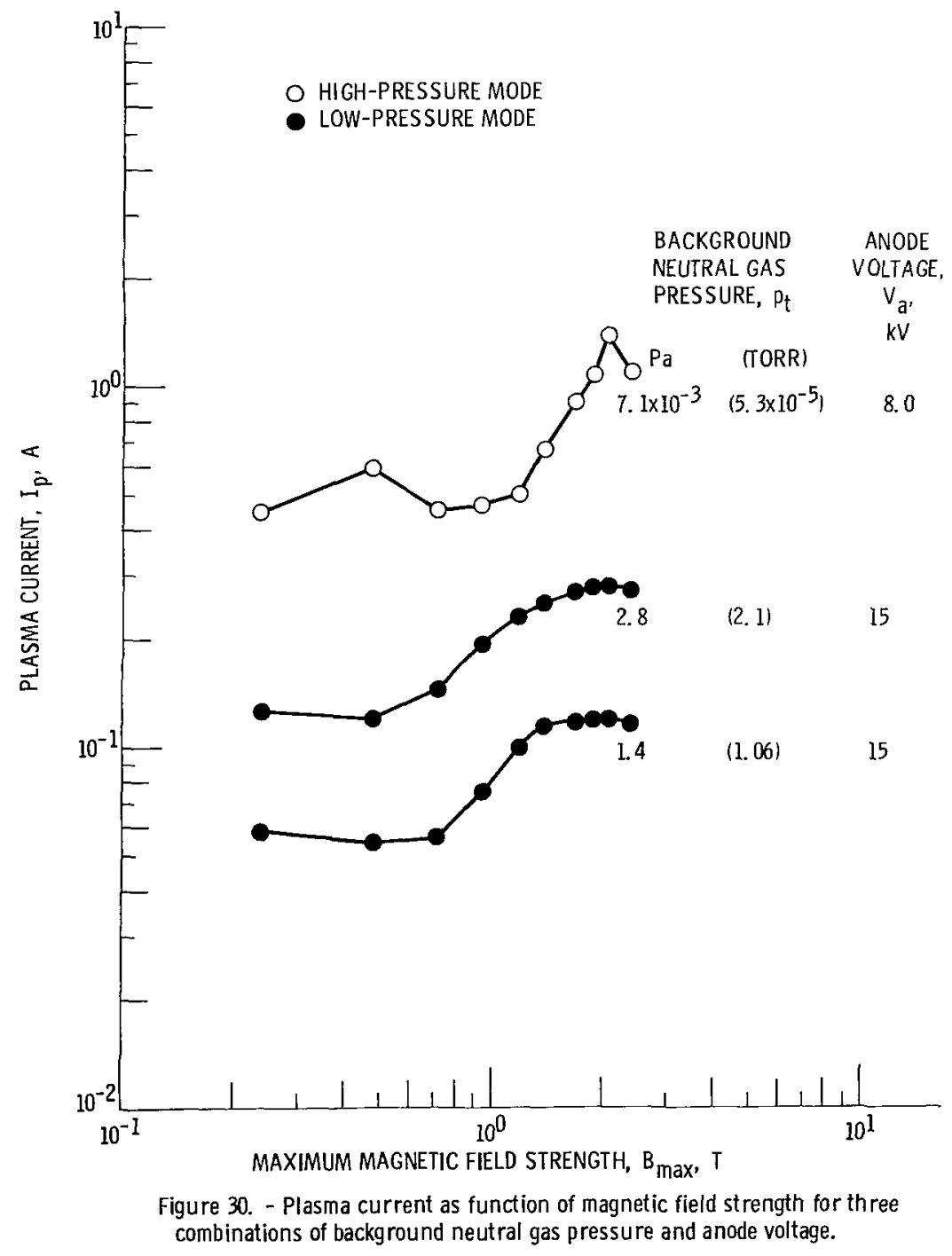


torus plasma, if charge neutrality is assumed, may be written

$$
\frac{d n_{e}}{d t}=n_{e} n_{0}\langle\sigma v\rangle_{n e}-\frac{n_{e}}{\tau_{c}}=0
$$

where $n_{0}$ is the neutral number density, $\langle\sigma v\rangle_{\text {ne }}$ is the ionization rate coefficient for electron-neutral ionization, and the time derivative on the left side vanishes in the steady state. Equation (5) assumes that ionization occurs by electron-neutral collisions only and that all parts of the plasma lose ions at an equal rate after an average residence time per particle of $\tau_{c^{*}}$. The average time required for an electron to produce an ionization is given by the ionization time $\tau_{\mathbf{i}}$

$$
\tau_{\mathrm{i}}=\frac{1}{\mathrm{n}_{0}\langle\sigma \mathrm{v}\rangle_{\mathrm{ne}}}=\tau_{\mathrm{c}}
$$

and is equal to the average ion residence time in the steady state. Plotting the average ion residence time as a function of magnetic field will then exhibit the manner in which the plasma containment depends on magnetic field.

Figure 31 is the average ion residence time in deuterium gas as a function of $\mathrm{mag}$ netic field over more than a factor of $\mathbf{1 0}$ variation in the latter quantity. Shown are data for two pressures at each magnetic field strength: one in the low-pressure mode of operation and one in the high-pressure mode of operation. The average ion residence

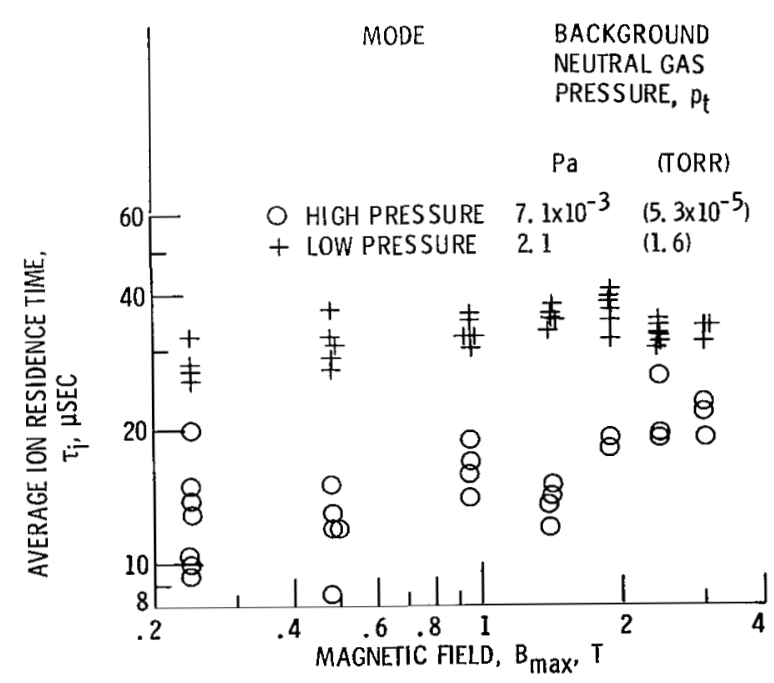

Figure 31. - Average ion residence time as function of maximum magnetic field strength for high-and lowpressure modes of operation. 
time is dependent very weakly, if at all, on the magnetic field strength in both the highand low-pressure modes of operation.

\section{EFFECT ON PLASMA CHARACTERISTICS OF VARYING THE}

\section{NUMBER OF ANODE RINGS}

The toroidal plasma was generated by applying a positive potential to all 12 anode rings, then only to the 6 even-numbered anode rings, and then to 3 anode rings located $120^{\circ}$ apart, and finally to a single anode ring. The anode rings not used were retracted from the discharge volume. The current-voltage curves for these cases in deuterium gas are shown in figures 32(a) and (b) for a single pressure in each mode and with the number of anode rings as a parameter. In general, the total anode current tended to increase as the number of anode rings decreased until the case of a single anode ring was reached. Figures 33(a) and (b) show similar data for helium gas, and the same general trend applies.

Spectroscopic measurements showed that the average ion residence time and number density both tended to increase as the number of anode rings decreased from 12 to 3 .

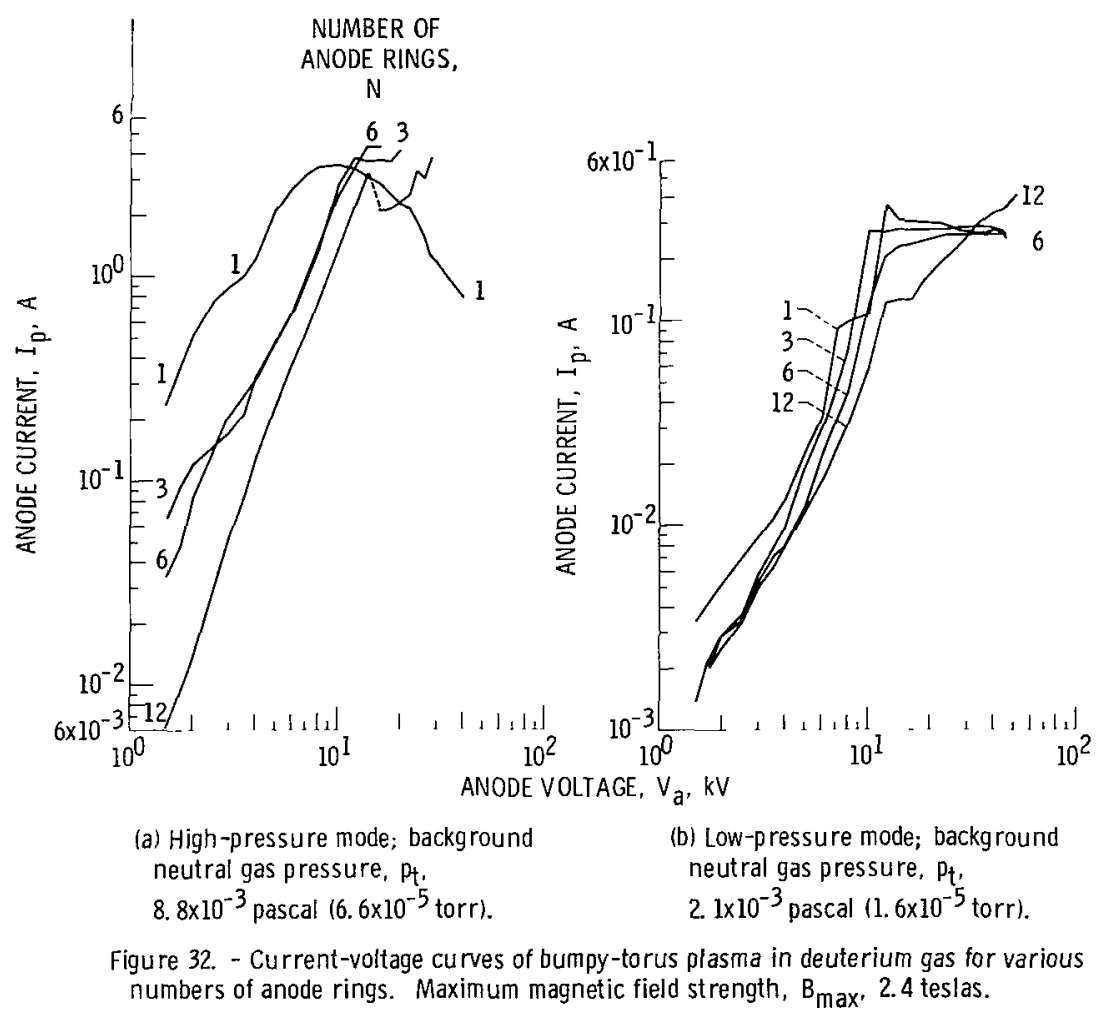




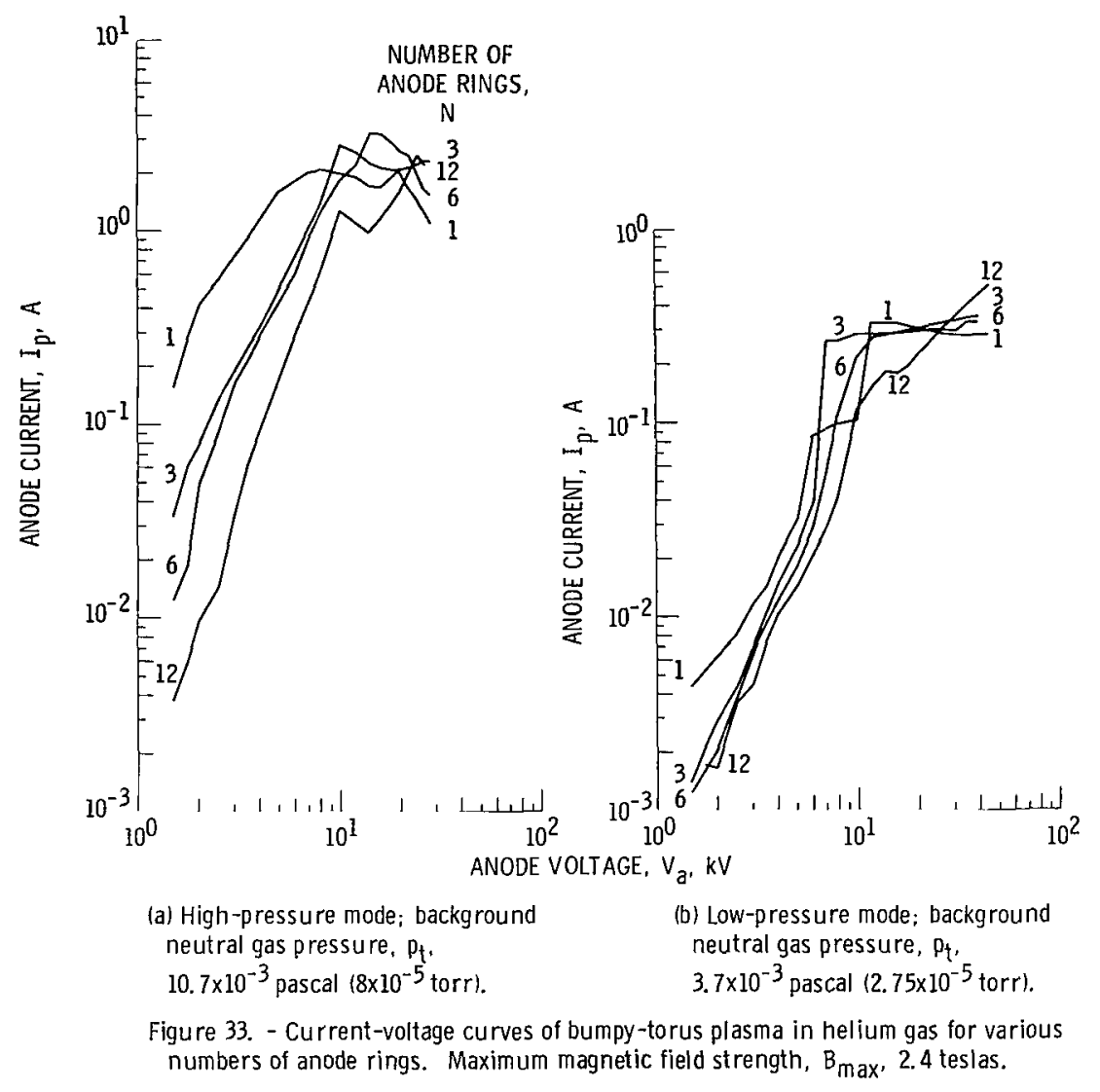

These results have been reported by Richardson (ref. 32). Figure 34 shows the relative electron number density on the axis as a function of the number of anode rings in the high- and low-pressure modes of operation. As the number of anode rings was reduced from 12 to 3 , the number density on the axis increased by approximately an order of magnitude in the high-pressure mode and by a factor of 3 in the low-pressure mode. The density resulting from operation with a single anode ring depended on the particular anode ring to which the voltage was applied. This may result from problems of alinement of the anode rings with respect to the magnetic axis.

The ion residence times $\tau_{c}$, which have been shown to be equal to the ionization times $\tau_{\mathfrak{i}}$, were calculated from equation (6) by using the spectroscopically determined radial profiles of electron temperature. Results are shown in figure 35 as a function of the number of anode rings $\mathrm{N}$ for both modes. Also indicated for each point is the corresponding mean electron temperature $\overline{\mathrm{T}}_{\mathrm{e}}$. The estimated error bars for the highpressure mode are large because of the steepness of the ionization rate curve at the lower electron temperatures. The increase in $\tau_{i}$ to 500 microseconds when going to $\mathbf{N}=3$ is quite pronounced, as well as the decrease when going to $N=1$. In the low- 


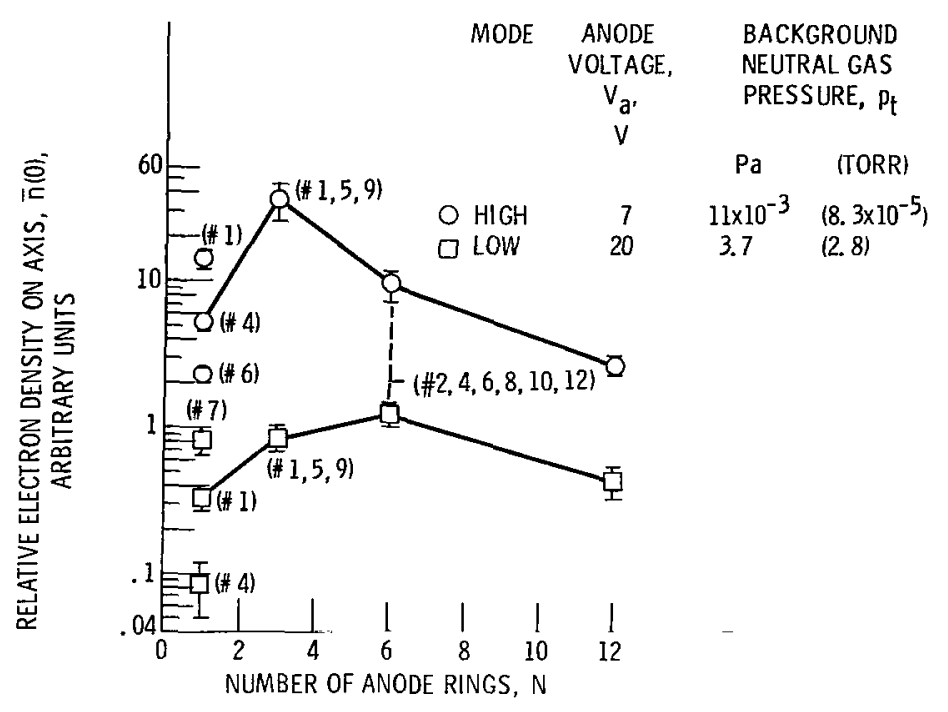

Figure 34. - Relative electron density on axis as function of number of anode rings for both modes of operation in helium gas. Maximum magnetic field strength, $B_{\max }, 2.4$ teslas.

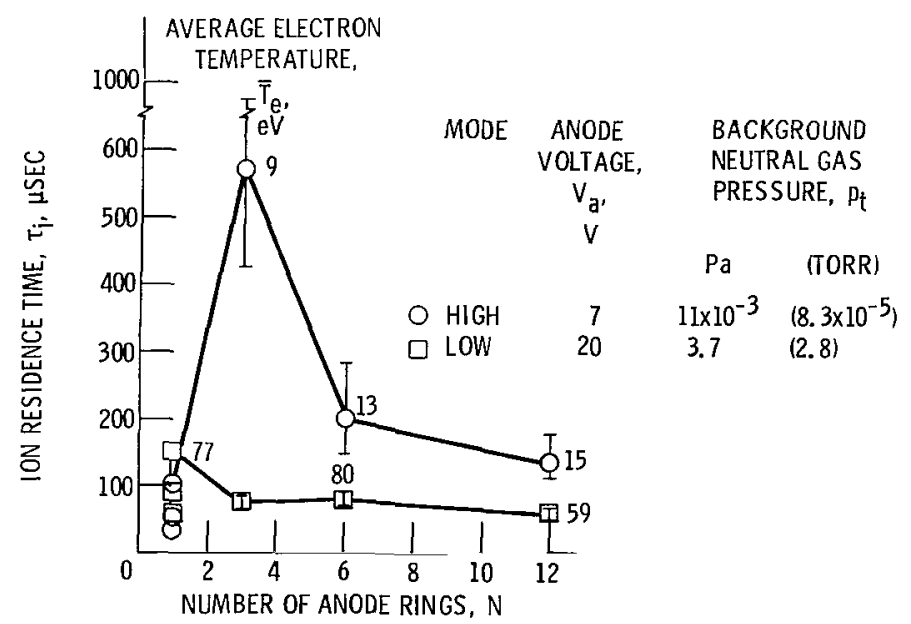

Figure 35. - Mean ion residence time as function of number of anode rings for both modes of operation in helium gas. Maximum magnetic field strength, $B_{\max }, 2.4$ teslas.

pressure mode, $\tau_{i}$ is essentially independent of $\mathrm{N}$ within the estimated error for $\mathrm{N}$ of 12,6 , and 3 . The error bars have been suppressed for the $N=1$ data because of the small scale and the crowding of points but are the same as for the other lowpressure-mode points. The scatter of the $\mathrm{N}=1$ points is apparently due to the pronounced difference in the discharge as reflected in the discharge current (figs. 32 
and 33), depending on which location is occupied by an anode. The energy loss times appropriate for Lawson parameter calculation are generally much shorter than $\tau_{i}$ in this helium discharge since charge-exchange collisions are more probable than ion loss.

It was pointed out previously that the current-voltage curves for the bumpy-torus plasma could be separated into three distinct regimes: the high-pressure-mode regime and high- and low-anode-voltage regimes of the low-pressure mode. Each of these three regimes has a characteristic slope of the constant-pressure current-voltage curves, defined by a power-law index $p$,

$$
\mathbf{I}_{\mathbf{p}} \sim \mathrm{V}_{\mathrm{a}}^{\mathrm{p}}
$$

It was of interest to determine whether the character of the current-voltage curves changed as the number of anode rings was varied. Current-voltage curves were taken in deuterium and helium gas at eight pressures and for symmetrical arrangements of $12,6,3$, and 1 anode rings. The slopes of the current-voltage curves were determined in the three regimes of operation. The only significant difference occurred for 12 anode rings. The high-pressure mode has a steeper slope for $\mathrm{N}=12$. Also the highanode-voltage regime of the low-pressure mode is proportional to $\mathrm{V}_{\mathrm{a}}$ for $\mathrm{N}=12$ but is independent of $\mathrm{V}_{\mathrm{a}}$ for fewer anode rings. This difference probably represents fundamentally different physical processes governing the radial transport when all 12 anode rings are present, relative to that occurring in the sectors with "missing" anode rings.

\section{DISCUSSION AND CONCLUSIONS}

A hot-ion, steady-state plasma has been generated in the bumpy-torus superconducting magnet facility by applying positive potentials as high as 50 kilovolts to the midplane electrode rings.

The magnet facility has met and exceeded its design magnetic field strength and has proved capable of more than 3 months of experimental running without breakdown. The use of superconducting magnets has made possible steady-state operation of the discharge, as well as unusually good visual and experimental access to the plasma for diagnostic measurements. Steady-state operation has allowed us to wait out the initial outgassing and impurity influx caused by turn-on of the plasma and subsequent plasmawall interactions. The operating conditions of this plasma are much more free of impurities than the initial turn-on phase.

The modified Penning discharge was successfully applied to heat a toroidal plasma. The ion kinetic temperatures observed, from 160 to 1785 electron volts in deuterium 
and 650 to 3300 electron volts in helium, are comparable to those observed in the simple magnetic mirror discharge under similar operating conditions. The plasma in the bumpy torus proved to be capable of operating over a very wide range of conditions, amounting to an order to magnitude or more for each of the independent parameters listed in table $\mathrm{I}$. The absolute values of the axial number density in helium were obtained by a spectroscopic procedure described in reference 32.

When the modified Penning discharge was operated in the simple magnetic mirror geometry, it was clear that the electrical circuit to the power supply could be completed by ions lost through the mirror throats to the grounded walls of the vacuum tank. In the bumpy-torus plasma, the anode rings collect electrons from the plasma, but the exact pathways and mechanisms by which ions are lost to the grounded coils and/or tank walls are not yet known.

The ion heating mechanism in the bumpy-torus plasma appears to be very similar if not identical to that previously observed in the modified Penning discharge in an axisymmetric magnetic mirror configuration (refs. 13 to 16). In both deuterium and helium plasmas, two distinct rotating spokes of ions and electrons as well as ion kinetic temperatures to several kilovolts were observed; and the ion energy distribution functions were Maxwellian for most operating conditions in both gases. In the bumpy-torus plasma, the ion energy distribution functions were very non-Maxwellian at low magnetic field strengths and became progressively more Maxwellian as the magnetic field strength increased.

At least two independent lines of evidence suggest that the radial transport of ions and electrons is virtually independent of magnetic field strength but depends on the background neutral gas density in an approximately linear manner. This observation is consistent with early investigations of the Penning discharge, in which it was observed that the anode current was proportional to the gas pressure (other parameters being held fixed), making it of interest as a vacuum gage. The independence of anode current and magnetic field strength tends to rule out either Bohm or classical diffusion as the controlling radial transport mechanism.

The NASA Lewis bumpy-torus plasma operates in a high-and a low-pressure mode. These modes of operation do not appear to be significantly affected by the type of gas used; and the mode transitions do not appear to be influenced by charge-exchange processes, metastable ion formation, or processes depending on whether the gas is monatomic or diatomic. The parameters that characterize the modes of operation are electron temperature and background neutral gas pressure. Lower electron temperatures characterize the high-pressure mode, higher electron temperatures characterize the low-pressure mode, and the high-pressure mode of operation is not observed below a certain threshold of pressure. The two modes of operation are further characterized by distinctly different radial number density profiles. The low-pressure mode exhibits a 
relatively flat radial density profile, and the high-pressure mode has a distribution peaked on the plasma axis.

Ten characteristic features of both modes of discharge operation are summarized in table IV. These features are characteristic of both deuterium and helium gas and held true with minor variations for magnetic field strengths from 0.5 to 3.0 teslas.

TABLE IV. - CHARACTERISTIC FEATURES OF THE TWO MODES OF DISCHARGE OPERATION

\begin{tabular}{|c|c|}
\hline High-pressure mode & Low-pressure mode \\
\hline High electron number density on axis & Low density on axis \\
\hline Density profiles peaked on axis & Density profiles flat or hollow \\
\hline Ionization through volume & Ionization in sheath \\
\hline $8 \leq \mathrm{T}_{\mathrm{e}} \leq 35 \mathrm{eV}$ & $35 \leq \mathrm{T}_{\mathrm{e}} \leq 200 \mathrm{eV}$ \\
\hline Much visible light & Plasma relatively dim \\
\hline Spokes obscure & Spokes well defined \\
\hline $\begin{array}{l}\text { High-radiofrequency noise back- } \\
\text { ground }\end{array}$ & Low-radiofrequency noise background \\
\hline $\begin{array}{l}\mathrm{E}_{\mathrm{r}}=0 \text { inside; } \mathrm{E}_{\mathrm{r}} \sim \mathrm{r} \text { in sheath } \\
\text { Minimum pressure threshold } \\
\mathrm{I}_{\mathrm{p}} \sim \mathrm{V}_{\mathrm{a}}^{3.0} \text { for } \mathrm{N}=12\end{array}$ & $\begin{array}{l}\mathrm{E}_{\mathrm{r}} \sim \mathrm{r} \text { across diameter } \\
\text { Mode exists at all pressures } \\
\mathrm{I}_{\mathrm{p}} \sim \mathrm{V}_{\mathrm{a}}^{2.0}, \mathrm{~V}_{\mathrm{a}} \text { for } \mathrm{N}=12\end{array}$ \\
\hline
\end{tabular}

The first eight characteristics suggest that the high-pressure mode is diffusion dominated and that ionization occurs throughout the volume by cooler electrons which are driven toward the plasma periphery by a radial density gradient. The first eight characteristics of the low-pressure mode are consistent with mobility-dominated radial transport. In this mode the electron energies must be near the maximum of the ionization rate coefficient in order to sustain the discharge, and the plasma interior is kept supplied by processes in the sheath. The spokes are prominent in the low-pressure mode because they are driven by electric fields that exist throughout the plasma volume and hence cause virtually the entire plasma to participate in the spoke dynamics. In the highpressure mode, the spokes are less prominent and their associated electrostatic potential fluctuations must compete with the background noise from the bulk of the plasma.

The ninth and tenth characteristics in table IV are anomalous in that they cannot readily be understood in association with, or as consequences of, the other eight characteristics listed. It is not at all clear, for example, why a threshold in background neutral gas pressure should exist in the high-pressure mode. Nor is it clear why variation in background neutral gas pressure will cause a mode transition, especially since the mean free path for neutral gas molecules is much greater than the plasma dimensions. Similarly, it is not obvious why the current-voltage curves should have different 
slopes in the two modes, which implies that fundamentally different physical processes are responsible for radial transport in the two modes.

The reduction of the number of anode rings used to generate the plasma seems to have a beneficial effect on the plasma number density and average ion residence time. Further investigation is required to optimize the alinement of the anode $r$ ings and the number of anode rings that maximize the plasma density and containment time for various operating conditions.

\section{Lewis Research Center,}

National Aeronautics and Space Administration,

Cleveland, Ohio, October 31, 1975, 506-25. 


\section{APPENDIX - SYMBOLS}

B magnetic field strength, $T$

$B_{\max } \quad$ maximum magnetic field strength, $T$

E electric field, $\mathrm{V} / \mathrm{m}$

$\mathrm{E}_{\mathrm{r}} \quad$ radial electric field, $\mathrm{V} / \mathrm{m}$

e electronic charge, $C$

$f_{j k}(v)$ apparent optical cross section for $j \rightarrow k$ transition

$\mathrm{f}_{l \mathrm{~m}}(\mathrm{v})$ apparent optical cross section for $l \rightarrow \mathrm{m}$ transition

$I_{j k} \quad$ intensity of spectral line due to $j \rightarrow k$ transition

$I_{l m} \quad$ intensity of spectral line due to $l-m$ transition

I $\quad$ anode current flowing to plasma, A

$\mathrm{m}$ minor azimuthal mode number

$\mathrm{N}$ number of anode rings

$n_{e} \quad$ electron number density

$\hat{\mathbf{n}}_{\mathrm{e}} \quad$ relative electron number density

$\hat{\mathrm{n}}_{\mathrm{e} 0} \quad$ arbitrary normalizing constant

$\mathrm{n}_{0} \quad$ neutral gas number density

$\mathrm{p}_{\mathrm{t}} \quad$ background neutral gas pressure in vacuum tank, $\mathrm{P}_{\mathrm{a}}$ (torr)

$R_{a} \quad$ effective anode resistance, $\Omega$

$R_{c} \quad$ equivalent cathode resistance, $\Omega$

$\mathrm{R}_{\mathrm{p}} \quad$ plasma resistance, $\Omega$

$r$ radius, $m$

$\mathrm{T}_{\mathrm{e}} \quad$ electron temperature, eV

$\mathrm{T}_{\mathbf{i}} \quad$ ion kinetic temperature, eV

t time, sec

$\mathrm{V}_{\mathrm{a}}$ anode voltage, $\mathrm{V}$

$v_{p} \quad$ plasma volume, $\mathrm{m}^{3}$

$\mathrm{v} \quad$ electron velocity, $\mathrm{m} / \mathrm{sec}$

$\mathrm{W}_{\mathrm{p}}$ measured input power to plasma, $\mathrm{W}$ 


$\begin{array}{ll}\mathrm{W}_{\mathrm{p} 0} & \text { calculated input power to plasma, } \mathrm{W} \\ \sigma_{j \mathrm{k}} & \text { maximum apparent cross section for } \mathrm{j} \rightarrow \mathrm{k} \text { transition } \\ \langle\sigma \mathrm{v}\rangle_{\text {ne }} & \text { electron-neutral ionization rate coefficient } \\ \tau_{\mathrm{c}} & \text { net charge residence time } \\ \tau_{\mathrm{ex}} & \text { charge-exchange time } \\ \tau_{\mathrm{i}} & \text { electron ionization time (defined by eq. }(6))\end{array}$




\section{REFERENCES}

1. Gibson, Gordon; Jordan, Willard C.; and Lauer, Eugene J.: Bumpy Torus. Phys. Rev. Lett., vol. 4, no. 5, Mar. 1960, pp. 217-219.

2. Gibson, Gordon; Jordan, Willard C. ; and Lauer, Eugene J.: Containment of Positrons in a Mirror Machine. Phys. Rev. Lett., vol. 5, no. 4, Aug. 1960, pp. 141-144.

3. Gibson, G.; Jordan, W. C. ; and Lauer, E. J.: Particle Behavior in Static, Axially Symmetric, Magnetic Mirror and Cusp Geometries. Phys. Fluids, vol. 6, no. 1, Jan. 1963, pp. 116-133.

4. Gibson, G. ; Jordan, W. C. ; and Lauer, E. J.: Particle Behavior in a Static, Asymmetric Magnetic Mirror Geometry. Phys. Fluids, vol. 6, no. 1, Jan. 1963, pp. 133-141.

5. Gibson, G.; et al.: Guiding Center Motion and Plasma Behavior in the Bumpy Torus. Phys. Fluids, vol. 7, no. 4, Apr. 1964, pp. 548-556.

6. Geller, R.: A Pulsed Toroidal P. I. G. Discharge. Appl. Phys. Lett., vol. 2, no. 11, May 1963, pp. 218-219.

7. Geller, R.: Experimental Results of a Bumpy Torus Discharge. Phys. Kev. Lett. vol. 10, no. 11, June 1963 , pp. $463-465$.

8. Fanchenko, S. D.; et al. : Turbulent Plasma Heating in a Torus. Phys. Rev Lett., vol. 21, no. 12, Sept. 1968, pp. 789-791.

9. Sprott, J. C.: Effect of Magnetic Field Errors on Confinement in Bumpy Tori. Phys. Fluids, vol. 16, no. 7, July 1973, pp. 1157-1159.

10. Roth, J. Reece; Rayle, Warren D.; and Reinmann, John J.: Technological Problems Anticipated in the Application of Fusion Reactors to Space Propulsion and Power Generation. NASA TM X-2106, 1970.

11. Roth, J. R.; Rayle, W. D.; and Reinmann, J. J.: Fusion Power for Space Propulsion. New Scientist, vol. 54, no. 792, Apr. 20, 1972, pp. 125-137.

12. Roth, J. Reece; Freeman, Donald C. ; and Haid, David A.: Superconducting Magnet Facility for Plasma Physics Research. Rev. Sci. Instru., vol. 36, no. 10, Oct. 1965, pp. 1481-1485.

13. Roth, J. Reece: Experimental Study of Spectral Index, Mode Coupling, and Energy Cascading in a Turbulent, Hot-Ion Plasma. Phys. Fluids, vol. 14, no. 10, Oct. 1971, pp. 2193-2202. 
14. Roth, J. Reece: Origin of Hot Ions Observed in a Modified Penning Discharge. Phys. Fluids, vol. 16, no. 2, Feb. 1973, pp. 231-236.

15. Roth, J. Reece: Hot Ion Production in a Modified Penning Discharge. IEEE Trans. on Plasma Sci., vol. PS-1, no. 1, Mar. 1973, pp. 34-45.

16. Roth, J. R. : Energy Distribution Functions of Kilovolt Ions in a Modified Penning Discharge. Plasma Physics, vol. 15, no. 10, Oct. 1973, pp. 995-1005.

17. Roth, J. Reece; et al.: Characteristics and Performance of a Superconducting Bumpy-Torus Magnet Facility for Plasma Research. NASA TN D-7353, 1973.

18. Roth, J. Reece; et al.: A 12-Coil Superconducting "Bumpy Torus" Magnet Facility for Plasma Research. Proc. 1972 Applied Superconductivity Conf., Inst. Elect. and Electronics Engrs., 1972, pp. 361-366.

19. Richardson, R. W.: Spectroscopic Results in Helium Gas from the NASA Lewis Bumpy Torus Plasma. Presented at Inst. Elect. and Elec. Engrs., First Intern. Conf. on Plasma Sci., Knoxville, Tenn., May 15-17, 1974.

20. Gerdin, Glenn A. : Spoke Wavenumbers and Mode Transitions in the NASA Lewis Bumpy Torus. Presented at Am. Phys. Soc. Sixteenth Annual Meeting, Albuquerque, N. Mex., Oct. 28-31, 1974.

21. Penning, F. M. ; and Nienhuis, K.: Construction and Applications of a New Design of the Phillips Vacuum Gauge. Phillips Tech. Review, vol. 11, no. 4, Oct. 1949, pp. 116-122.

22. Gerdin, Glenn A.: Radio Frequency Studies in the NASA Lewis Bumpy Torus. Presented at Inst. Elect. Elec. Engrs. First Intern. Conf. on Plasma Sci., Knoxville, Tenn., May 15-17, 1974.

23. Valckx, F. P. G.: Electrostatic Analyzer for the Detection of Fast Neutral Particles. NASA TTF-11458, 1968.

24. Englert, Gerald W. ; Reinmann, John J.; and Lauver, Milton R.: Guiding Center Model to Interpret Neutral Particle Analyzer Results. Presented at Inst. Elect. Elec. Engrs. First Intern. Conf. on Plasma Sci., Knoxville, Tenn., May 15-17, 1974.

25. Sovie, Ronald J.: Spectroscopic Determination of Electron Temperature and Percentage Ionization in a Helium Plasma. Phys. Fluids, vol. 7, no. 4, Apr. 1964, pp. 613-614.

26. Latimer, I. D.; Mills, J. I. ; and Day, R. A. : Refinements in the Helium Line Ratio Technique for Electron Temperature Measurement and Its Application to the Precursor. J. Quant. Spectr. Rad. Trans., vol. 10, 1970, pp. 629-635. 
27. Jobe, John D.; and St. John, Robert M.: Absolute Measurements of the $2^{1} P$ and $2^{3}$ P Electron Excitation Cross Sections of Helium Atoms. Phys. Rev., vol. 164, no. 1, Dec. 1967, pp. 117-121.

28. Kay, R. B.; and Showalter, J. G.: Exchange Excitation in Helium: Excitation Functions for the $4^{3} \mathrm{~S}, 3^{3} \mathrm{P}$, and $3^{3}$ D Levels. Phys. Rev. A., vol. 3, no. 6, June 1971, pp. 1998-2006.

29. St. John, Robert M. ; and Lin, Chun C.: Production of Excitation and Ionization in Helium by Single-Electron Impact. J. Chem. Phys., vol. 41, no. 1, July 1964, pp. 195-197.

30. Moussa, H. R. Moustafa; and De Heer, J. F.: Excitation of Helium to Excited Ion States by Electron and Proton Impact. Physica, vol. 36, 1967, pp. 646-654.

31. Lochte-Holtgreven, W., ed.: Plasma Diagnostics. North Holland Publ. Co., 1968, pp. 184-186.

32. Richardson, R. W.: Effect of Anode Ring Arrangement on the Spectroscopic Characteristics of the NASA Lewis Bumpy Torus Plasma. Presented at Am. Phys. Soc. Sixteenth Annual Meeting, Albuquerque, N. Mex., Oct. 28-31, 1974. 
"The aeronautical and space activities of the United States shall be conducted so as to contribute ... to the expansion of buman knowledge of phenomena in the atmospliere and space. The Administration shall provide for the widest practicable and approprate dissemination of information concerning its activities and the results thereof."

-National aeronautics and Space ACt of 1958 .

\section{NASA SCIENTIFIC AND TECHNICAL PUBLICATIONS}

TECHNICAL REPORTS: Scientific and technical information considered important, complete, and a lasting contribution to existing knowledge.

TECHNICAL NOTES: Information less broad in scope but nevertheless of importance as a contribution to existing knowledge.

TECHNICAL MEMORANDUMS:

Information receiving limited distribution because of preliminary data, security classification, or other reasons. Also includes conference proceedings with either limited or unlimited distribution.

CONTRACTOR REPORTS: Scientific and technical information generated under a NASA contract or grant and considered an important contribution to existing knowledge.
TECHNICAL TRANSLATIONS: Information published in a foreign language considered to merit NASA distribution in English.

SPECIAL PUBLICATIONS: Information derived from or of value to NASA activities. Publications include final reports of major projects, monographs, data compilations, handbooks, sourcebooks, and special bibliographies.

\section{TECHNOLOGY UTILIZATION}

PUBLICATIONS: Information on technology used by NASA that may be of particular interest in commercial and other non-aerospace applications. Publications include Tech Briefs, Technology Utilization Reports and Technology Surveys.

Defails on the availability of these publications may be obtained from:

SCIENTIFIC AND TECHNICAL INFORMATION OFFICE 